\title{
The Sample Complexity of Auctions with Side Information*
}

\author{
Nikhil R. Devanur \\ Microsoft Research, \\ Redmond, USA \\ nikdev@microsoft.com
}

\author{
Zhiyi Huang ${ }^{\dagger}$ \\ The University of Hong Kong, \\ China \\ zhiyi@cs.hku.hk
}

\author{
Christos-Alexandros \\ Psomas \\ UC Berkeley, USA \\ alexpsomi@cs.berkeley.edu
}

\begin{abstract}
Traditionally, the Bayesian optimal auction design problem has been considered either when the bidder values are i.i.d, or when each bidder is individually identifiable via her value distribution. The latter is a reasonable approach when the bidders can be classified into a few categories, but there are many instances where the classification of bidders is a continuum. For example, the classification of the bidders may be based on their annual income, their propensity to buy an item based on past behavior, or in the case of ad auctions, the click through rate of their ads. We introduce an alternate model that captures this aspect, where bidders are a priori identical, but can be distinguished based (only) on some side information the auctioneer obtains at the time of the auction.

We extend the sample complexity approach of Dhangwatnotai et al. and Cole and Roughgarden to this model and obtain almost matching upper and lower bounds. As an aside, we obtain a revenue monotonicity lemma which may be of independent interest. We also show how to use Empirical Risk Minimization techniques to improve the sample complexity bound of Cole and Roughgarden for the nonidentical but independent value distribution case.
\end{abstract}

\section{Categories and Subject Descriptors}

F.2.0 [Theory of Computation]: Analysis of Algorithms and Problem Complexity.General

\section{General Terms}

Algorithms, Economics

${ }^{*}$ A full version of this paper is available at arXiv 6. Part of this work was done while the authors were visiting the Simons Institute for the Theory of Computing.

†Supported by an RGC grant HKU27200214E.

$\ddagger_{\text {This research was supported by NSF grants CCF0964033 }}$ and CCF1408635, and by Templeton Foundation grant 3966, as well as the Leventis Foundation.

Permission to make digital or hard copies of all or part of this work for personal or classroom use is granted without fee provided that copies are not made or distributed for profit or commercial advantage and that copies bear this notice and the full citation on the first page. Copyrights for components of this work owned by others than ACM must be honored. Abstracting with credit is permitted. To copy otherwise, or republish, to post on servers or to redistribute to lists, requires prior specific permission and/or a fee. Request permissions from Permissions@acm.org.

Copyright is held by the owner/author(s). Publication rights licensed to ACM.

STOC'16, June 19-21, 2016, Cambridge, MA, USA

ACM. 978-1-4503-4132-5/16/06...\$15.00

http://dx.doi.org/10.1145/2897518.2897553

\section{Keywords}

Myerson's auction, sample complexity

\section{INTRODUCTION}

Myerson's 16 theory of optimal auctions has been (deservingly) much celebrated. When bidders' values are independently and identically distributed (iid), it gives the beautiful conclusion that a second price auction with a reserve price is revenue optimal among all incentive compatible and individually rational auction formats. When the bidders are distinguishable, via a distinct value distribution for each bidder, the theory still characterizes the optimal auction, even though it is not as simple as in the iid case.

The non-identical but independent distribution (non-iid) model could be applicable in practice when bidders can be classified into a few different categories, and market research provides a distribution for each of these. The starting point for this paper is the observation that often, the classification of bidders is a continuum, rather than discrete. Suppose for instance that the auctioneer knows the incomes of all the bidders. It is reasonable to assume that bidders with higher incomes in general have higher valuations and thus use this information in the auction design. Similarly the auctioneer could use knowledge about the bidders' past purchasing behavior to distinguish between them. The same observation applies for a seller who simply offers an item at a posted price, which is equivalent to an "auction with a single agent".

In this paper, we propose a model where the side information that can be used to distinguish between the bidders comes from a continuum. A priori, bidders are identical: all bidders draw two numbers, a value and a "signal", iid from a joint probability distribution. The signal is a real number in $[0,1]$ that captures the side information that the auctioneer has about the bidder. We assume that the marginal distribution of the values conditioned on the signal is monotone in the signal, in the order of first order stochastic dominance.

Even when the bidder categories are discrete ${ }^{1}$ it is more natural that there is a distribution over categories. If bidders from some categories appear more often than others, then it is more important that the auction does the right thing for these categories. Also, it is more natural that this is represented in the samples as well, so the number of samples for each category would be different if you collected samples at random from the population. Further, it is very common to have a total ordering on the categories, where bidders in

\footnotetext{
${ }^{1}$ Note that our model can handle discrete categories as well, where the signal is just an encoding of the categories.
} 
the higher categories have higher values, and this structure could be exploited. (See 14, 3 for example.) Our model incorporates these aspects of the real world and hence is a better fit for many practical applications.

\section{Ad Auctions.}

Billions of auctions are run each day for ads, for search and display advertising. Setting reserve prices in these auctions has received a lot of attention 10, 21, 18, 17, and has been one of the prime applications of Myerson's theory 17]. Common techniques in practice include using machine learning algorithms, to map features of an auction into one of few categories, and use the corresponding reserve price. These settings might benefit from a "continuous categorization" ${ }^{2}$ where the machine learning algorithms map the features to a real number instead. This would correspond exactly to our model, with this number as the signal. One such number might already be calculated: it has been observed that the click-through rates are positively correlated with bids/values, so these rates can be used as the signals. Such a phenomenon, of using the click-through rates to differentially charge bidders already happens currently, although indirectly, through the practice of "squashing" 13 .

\section{Sample Complexity.}

When the joint probability distribution over values and signals is completely known to the auctioneer, Myerson's theory readily extends to this case: simply run Myerson's auction on the conditional value distributions. The question that we consider in this paper is that of sample complexity: how many samples from the distribution are necessary/sufficient to approximate the optimal auction?

The sample complexity approach to revenue maximization $7,5,12,15$ assumes that we are given access to samples from the value distribution and measures the number of samples that are necessary/sufficient to approximate the optimal auction. This study is motivated by the observation that the source of priors is really past data, both in principle and in practice 17. The goal has been to put such practices on a sound theoretical footing, by identifying principled approaches and precisely quantifying the value of data.

Dhangwatnotai et al. 7] initiated this line of research, by giving almost tight bounds for the case of a single agent, which was later extended to multiple agents ( with nonidentical but independent regular distributions ) by Cole and Roughgarden [5]. In this paper we improve the upper bound in their 5] model, which we'll refer to as the non-iid model, from $\tilde{O}\left(n^{10} \epsilon^{-7}\right)$ to $\tilde{O}\left(n \epsilon^{-4}\right)$. A detailed discussion of the upper and lower bounds for different variants, and comparisons with other related work such as 15, 12 is in Section 2.2

\section{Techniques.}

The improvement in the sample complexity upper bounds in the non-iid model comes from taking a PAC learning/ Empirical Risk Minimization (ERM) approach. We first show how to discretize the value space while losing only an $O(\epsilon)$ fraction of the revenue. This then allows us to bound the number of different mechanisms that we need to con-

\footnotetext{
${ }^{2}$ The techniques used are mostly from continuous optimization, with extra effort required to map these into discrete categories.
}

sider. Then we use a concentration inequality to argue that for each mechanism in this class, its revenue on the sample closely approximates its actual revenue from the distribution. Thus, using the best auction on the sample suffices.

As an important part of this approach we prove a new concentration inequality that might be of independent interest. Consider the revenue of a mechanism with $n$ agents in the non-iid model. Consider a matrix of values, each drawn independently, with the values in column $i$ drawn from the distribution corresponding to agent $i$. Chernoff bounds tells us that the revenue of the mechanism averaged over the row vectors of this matrix is close to its expected revenue w.r.t the distribution. Our concentration inequality gives the same conclusion for the revenue of the mechanism w.r.t. the product of the empirical distributions over each column of the matrix. Due to this, instead of finding the optimal auction for a correlated distribution, it is sufficient to find the optimal auction for a product distribution, a computationally easy task.

The model with side information, which we'll refer to as the Signals model, presents the following new conceptually important challenge: we cannot rely on having seen samples with exactly the same signals as the participants in the auction. This requires us to be able to interpolate using samples from different distributions, rather than use samples from the same distribution as in the non-iid model ${ }^{3}$ We do this by using just the right number of signals in the sample immediately below the actual signal of an agent, and run a version of the Empirical Myerson auction. The approximation factor is proved via a careful charging argument. Our bounds depend on the following quantity: suppose that an $\epsilon$ fraction of the revenue is contributed by the top $q(\epsilon)$ fraction (in probability mass) of signals. For instance, when there is a single agent, $O\left(\log \left(\frac{1}{\epsilon}\right) q(\epsilon)^{-1} \epsilon^{-3}\right)$ samples are sufficient and $\Omega\left(\log \left(\frac{1}{q(\epsilon)}\right) \epsilon^{-4}\right)$ are necessary ${ }^{4}$

An essential component of interpolating from different distributions is revenue monotonicity. Consider the optimal auction $M$ for a given product distribution $\mathbf{D}$. If $M$ is run on values that come from a higher (component wise first order stochastic dominating) distribution, does it get a higher revenue? Surprisingly, as far as we know, this fundamental question about optimal auctions was unanswered prior to this paper; we resolve this in the affirmative. Another application of this property is in devising mechanisms when only certain statistics (such as the medians) of the value distributions are known (1 $) t^{5}$ The proof of this property is indirect; we first reduce this to a weaker property, namely monotonicity of the optimum revenue (over a larger class of distributions), using Sion's minimax theorem 19]. We then show monotonicity of the optimum revenue using convexity of the optimum revenue in the space of distributions.

\footnotetext{
${ }^{3}$ This introduces additional difficulties such as that convex combinations of regular distributions are not regular, e.g., see 20.

${ }^{4}$ This is not a contradiction since $q(\epsilon)$ is always at most $\epsilon$ and $x \log (1 / x)$ is a monotone increasing function for small enough $x$.

${ }^{5} \mathrm{~A}$ natural mechanism to consider in such a setting is the optimal mechanism w.r.t. the minimal distribution with the given statistics. Given the value of the median $\mu$, the distribution which is 0 or $\mu$ with probability $\frac{1}{2}$ each is first order stochastically dominated by all other distributions with median $\mu$.
} 


\section{Remark.}

Direct approaches seem to fail here. Consider the following example, where increasing one component distribution first increases the revenue, and then increasing another component decreases the revenue, of an optimal mechanism for the original distribution. There are two bidders: bidder 1 has value distribution $U[0,100]$ and bidder 2 has distribution $U[0,1]$. The virtual value functions are $\phi_{1}=2 v-100$ and $\phi_{2}=2 v-1$ respectively. If bidder 1's distribution is replaced by $U[50,100]$, the revenue strictly increases, since the probability of allocating the item to her (for a high price) increases. In fact bidder 1 always gets the item, except when she has a value in $[50,50.5]$ and bidder 2 has a value in $[0.5,1]$ with higher virtual value. When this occurs the item is sold to bidder 2 for a low price. The probability of this event strictly increases when bidder 2's distribution is replaced with a point mass at 1 , and thus the revenue slightly decreases. The net effect is still an increase in the revenue, as guaranteed by our theorem. This also shows that revenue monotonicity is not true w.r.t any mechanism, i.e., if you consider an arbitrary mechanism and consider its revenue on two distributions where one stochastically dominates the other, then the revenue of the dominating distribution need not be higher.

For the lower bounds, we extend the instances used by Huang et al. [12, by packing scaled copies of them among the conditional distributions for different signals. We can show that in order to achieve high revenue overall it is necessary to achieve high revenue in most of the conditionals. Moreover, in order to get good revenue from any conditional (even with exact knowledge of the rest) a reduction to classification shows that $\epsilon^{-3}$ samples are necessary; the bound follows.

\section{Extensions.}

Our results can be extended to more general single parameter families of auction environments. We can also get better upper bounds for the case of $n$ agents when we are required to optimize over a smaller class of auctions, such as VCG with reserve prices. We discuss these extensions in Section 6

\section{Other Related Work.}

Elkind 9] also considers a learning question very similar to the sample complexity line of work $7,5,12,15$, in the presence of an oracle that returns the expected profit of a given mechanism, for distributions with finite support. This line of work is also close in spirit to Balcan et al. 2], who use PAC learning techniques to design prior-free auctions in a general setting. In fact, Huang et al. 12 note that the results of Balcan et al. 2] can be used to deduce sample complexity bounds in the iid model. In addition to the lower bound of $\Omega\left(\epsilon^{-3}\right)$ for regular distributions, Huang et al. 12 also show tight bounds of $\tilde{\Theta}\left(\epsilon^{-3 / 2}\right)$ for MHR distributions. While all these results are for single parameter environments, Dughmi et al. 8. show an exponential lower bound for a multi parameter setting.

\section{Future Directions.}

Much of the auction theory since Myerson, in particular in the algorithmic game theory literature, is devoted to cases where the distributions are not known. One can ask several "simple vs. optimal" questions in the side information setting, such as an extension of the famous result of Bulow and Klemperer [4]: how much does the market size have to increase for the Vickrey auction to outperform the optimal auction. Another interesting direction is to ask for the design of a prior-independent mechanism in this model, which might be easier than a similar attempt in the prior-free setting by Leonardi and Roughgarden [14] and Bhattacharya et al. 3. Finally, extending this model to align it even closer with how machine learning algorithms are used, by directly considering high dimensional signal space is a really exciting direction.

\section{PRELIMINARIES AND MAIN RESULTS 2.1 Preliminaries}

\section{Auctions.}

A single item is auctioned to $n$ bidders; the value of bidder $i$ for the item is $v_{i}$. From the revelation principle, it is sufficient to consider direct revelation mechanisms: a mechanism $M$ is a pair of functions $(x, p)$, both taking as input a reported value profile $\mathbf{v}=\left(v_{1}, \ldots, v_{n}\right)$. The allocation function $x$ has for each bidder $i$, a component $x_{i}$ that represents her probability of winning the item. Similarly the payment function $p$ has component $p_{i}$ for the payment of bidder $i$. The utility of bidder $i$ is $v_{i} x_{i}-p_{i}$.

A mechanism is dominant strategy incentive compatible (DSIC) if the utility of a bidder $i$ is maximized, no matter what the other agents report, by reporting her true value. A mechanism is individually rational (IR) if the utility of all bidders is non-negative, for all valuation profiles.

The objective we consider in this paper is the revenue of a mechanism, when values are drawn from a given probability distribution. Let $\operatorname{REv}(M, \mathbf{D})$ denote the expected revenue of mechanism $M$ when $\mathbf{v}$ is drawn from distribution $\mathbf{D}$ :

$$
\operatorname{REV}(M, \mathbf{D}) \stackrel{\text { def }}{=} \mathbb{E}_{\mathbf{v} \sim \mathbf{D}}\left[\sum_{i} p_{i}(\mathbf{v})\right] .
$$

Throughout the paper we only consider DSIC and IR mechanisms, and therefore omit qualifying mechanisms as DSIC and IR. The mechanism that maximizes $\operatorname{REV}(\cdot, \mathbf{D})$ for a given distribution $\mathbf{D}$, among all DSIC and IR mechanisms, is called the optimal auction, and its revenue is denoted by Opt (D).

\section{Myerson's Optimal Auction.}

Myerson 16 characterizes the optimal auction for product distributions $\mathbf{D}=D^{1} \times \cdots \times D^{n}$. The optimal auction is simple to describe under the assumption that each $D^{i}$ is regular, which means that the virtual value function $\phi_{i}(v) \stackrel{\text { def }}{=}$ $v-\frac{1-F_{i}(v)}{f_{i}(v)}$ is monotonically non decreasing, where $f_{i}$ is the density and $F_{i}$ is the CDF of $D^{i}$. For this case, Myerson's optimal auction allocates the item to the bidder with the highest virtual value (breaking ties arbitrarily). The winner pays

$$
\begin{aligned}
p_{i} & \stackrel{\text { def }}{=} \min \left\{p: \forall j \neq i, \phi_{i}(p) \geq \phi_{j}\left(v_{j}\right), \text { and } \phi_{i}(p) \geq 0\right\} \\
& =\max _{j \neq i}\left\{\phi_{i}^{-1}\left(\phi_{j}\left(v_{j}\right)\right)\right\} \cup\left\{\phi_{i}^{-1}(0)\right\}
\end{aligned}
$$

\section{Sample Complexity.}

Dhangwatnotai et al. 7] and Cole and Roughgarden [5] introduced a sample complexity approach to the design of op- 
timal auctions. Assume that the distribution D is unknown to the auctioneer, who instead has to design a mechanism $M$ with only access to data in the form of $m$ i.i.d. samples $s^{(1)}, \ldots, s^{(m)}$ drawn from $\mathbf{D}$. The mechanism $M$ is then run on a fresh vector of values (the real input) drawn from $\mathbf{D}$.

The sample complexity of a given mechanism $M$ against a given class of distributions is specified as a function of $\epsilon \in[0,1]$, and is defined to be the smallest number of samples $m$ such that for all distributions $\mathbf{D}$ in the given class

$$
\mathbb{E}_{s^{(1)}, \ldots, s}(m) \sim \mathbf{D}[\operatorname{REV}(M, \mathbf{D})] \geq(1-\epsilon) \operatorname{OpT}(\mathbf{D}) .
$$

Here it is implicit that the mechanism $M$ depends on the samples $s^{(1)}, \cdots, s^{(m)}$.

\section{Auctions with Side Information.}

In this paper, we introduce a model where the auctioneer has some information correlated with the bidders' values. We assume that bidder $i$ has a value-"signal" pair $\left(v_{i}, \sigma_{i}\right)$, drawn from a joint distribution $\mathcal{D}$. As before, $v_{i}$ is her valuation for the item and $\sigma_{i} \in[0,1]$ is a signal observed by the auctioneer, e.g., her annual income, her propensity to buy an item based on past behavior, or in case of ad auctions the click-through rate of her ad. For a given signal $\sigma$, we denote the conditional distribution over values by $D^{\sigma}$. It is convenient to think of $\mathcal{D}$ as a family of 1-dimensional distributions $\left\{D^{\sigma}: \sigma \in[0,1]\right\}$, along with the marginal distribution over $\sigma$, given by the $\operatorname{cdf} F_{\mathcal{D}, \sigma}$. We assume throughout this paper that $\mathcal{D}$ is such that if $\sigma_{i}>\sigma_{j}$, then $D^{\sigma_{j}}$ is first order stochastically dominated by $D^{\sigma_{i}}$ (denoted by $D^{\sigma_{i}} \succeq D^{\sigma_{j}}$ ). The mechanism now has as input the actual signals, in addition to the reported values.

We extend the sample complexity approach to this model: the auctioneer first observes $m$ i.i.d. value-signal pairs sampled from the given distribution, which can be used to design a mechanism. The sample complexity will as before depends on $\epsilon \in(0,1)$ (among other parameters), and is defined as the minimum number of samples $m$ required to achieve a $1-\epsilon$ fraction of the optimal revenue $\operatorname{OpT}^{\text {signals }}(\mathcal{D})$, defined as

$$
\operatorname{OpT}^{\text {signals }}(\mathcal{D}) \stackrel{\text { def }}{=} \mathbf{E}_{\sigma_{1}, \ldots, \sigma_{n} \sim F_{\mathcal{D}, \sigma}}\left[\operatorname{OpT}\left(D^{\sigma_{1}} \times \cdots \times D^{\sigma_{n}}\right) .\right]
$$

The Signals model is incomparable to the non-iid model. Note that in this model, the bidders are a priori identical, but become non identical once the signals are observed. This allows a mechanism to be a priori symmetric, and distinguish between the bidders based only on the signal. Also in this model, the samples may contain entirely different signal values than the actual signals. Further, there is a distribution over signals themselves which affects the definition of $\mathrm{OPT}^{\text {signals }}(\mathcal{D})$. Both of the models however generalize the case of i.i.d value distributions. We call a distribution $\mathcal{D}$ regular if each $D^{\sigma}$ is regular.

\subsection{Main Results}

Our results come in two flavors: we improve the upper bound of Cole and Roughgarden [5], as well as show lower and upper bounds for the Signals model. We significantly lower the gap between the upper and lower bounds from [5], which were $\Omega\left(n \epsilon^{-1}\right)$ and $\tilde{O}\left(n^{10} \epsilon^{-7}\right)$ respectively. For the single agent case, Huang et al. 12] showed a lower bound of $\Omega\left(\epsilon^{-3}\right)$.

Definition 1 (SMALL-TAIL In Signal SPACE). Given a function $q:[0,1] \rightarrow[0,1]$, say that a distribution $\mathcal{D}$ has a q-bounded tail in the signal space if

$$
\begin{gathered}
\mathbf{E}_{\sigma_{1}, \ldots, \sigma_{n} \sim F_{\mathcal{D}, \sigma}}\left[\mathbb{1}\left\{\forall i, F_{\mathcal{D}, \sigma}\left(\sigma_{i}\right) \leq 1-q(\epsilon)\right\} \mathrm{OPT}\left(D^{\sigma_{1}} \times \cdots \times D^{\sigma_{n}}\right)\right] \\
\geq(1-\epsilon) \mathrm{OPT}^{\text {signals }}(\mathcal{D})
\end{gathered}
$$

Table 1 summarizes the main results.

For the case of a single agent, the Signals model has an upper bound that is larger by a factor of $1 / q(\epsilon)$, while the lower bound is larger by a factor of $\log (1 / q(\epsilon)) \epsilon^{-1}$, and $q(\epsilon)$ is always no larger than $\epsilon$. It is to be expected that the Signals model requires more samples, since we need to cover the entire spectrum of different $D^{\sigma} \mathrm{s}$. At least a linear dependence on $q(\epsilon)$ seems necessary, since we need at least those many samples to even see a single $\sigma$ in the top $q(\epsilon)$ quantile, which contribute an $\epsilon$ fraction of revenue.

With $n$ agents, note that one sample in the non-iid model is actually $n$ values, whereas one sample in the Signals model is only one value. Taking this into account, the upper bounds still differ by a factor of $1 / q(\epsilon)$. We currently don't have any lower bounds for the Signals model, since in this model, having more agents could actually help! Closing the gap between the bounds, or parameterizing the bounds using other reasonable quantities are interesting open questions.

Morgenstern and Roughgarden [15] consider the sample complexity question à la Cole and Roughgarden [5], but restricted to bounded support distributions. The support is assumed to lie in $[1, H]$, for some fixed value of $H$, therefore their bounds are not directly comparable to ours. They show an upper bound of $O\left(n H^{3} \epsilon^{-3}\right)$. They use the technique of bounding the pseudo-dimension, from statistical learning theory, as compared to our more elementary technique of building an $\epsilon$-net. Both approaches are similar in spirit; however, their result also generalizes to single parameter environments beyond single item auctions.

\section{Revenue Monotonicity.}

We say that a product distribution $\mathbf{D}=\Pi_{i} D^{i}$ componentwise stochastically dominates a product distribution $\mathbf{D}^{\prime}=$ $\Pi_{i} D^{i^{\prime}}$ (and denote it by $\mathbf{D} \succeq \hat{\mathbf{D}}$ ) if for each $i$, we have that $D^{i} \succeq D^{i^{\prime}}$. We prove the following theorem which might be of independent interest:

Theorem 1. [Revenue Monotonicity] Let $\mathbf{D}_{0}$ be a product distribution with finite support. There exists $M_{0}$ which is an optimal auction for $\mathbf{D}_{0}$ such that for all finite support distributions $\mathbf{D} \succeq \mathbf{D}_{0}$,

$$
\operatorname{ReV}\left(M_{0}, \mathbf{D}_{0}\right) \leq \operatorname{REV}\left(M_{0}, \mathbf{D}\right) .
$$

REMARK 1. All optimal auctions are virtual welfare maximizers, identical up to tie breaking rules. We can make the optimal auction for a product distribution $\mathbf{D}_{0}$ unique as follows: add an infinitesimal $\epsilon_{i}$ to all values in the (finite) support of $\mathbf{D}_{0}^{i}$, such that $\epsilon_{i}>\epsilon_{i+1}$. In the new product distribution the revenue is the same, but the only optimal tie breaking rule is lexicographical.

\section{Concentration Inequality.}

We prove the following concentration inequality, which is useful in showing that the revenue of a mechanism on a given product distribution is close to its revenue on the empirical product distribution. This should be of independent interest. 


\begin{tabular}{|c|c|c|c|c|}
\hline Model & $\begin{array}{l}\text { Number } \\
\text { of agents }\end{array}$ & Distributions & Lower Bound & Upper bound \\
\hline non-iid model & 1 & Regular & $\Omega\left(\epsilon^{-3}\right) \quad 12$ & $O\left(\epsilon^{-3} \log \frac{1}{\epsilon}\right) \overline{7}$ \\
\hline non-iid model & $n$ & Regular & $\Omega\left(\max \left\{n \epsilon^{-1}, \epsilon^{-3}\right\}\right)[5,12$ & $\tilde{O}\left(n \epsilon^{-4}\right)$ (Sec. 4 ) \\
\hline Signals model & 1 & Regular, $q$ bounded tail & $\Omega\left(\epsilon^{-4} \log \left(\frac{1}{q(\epsilon)}\right)\right)$ (Sec. $5.3 p$ & $O\left(q(\epsilon)^{-1} \epsilon^{-3} \log \left(\frac{1}{\epsilon}\right)\right)$ (Sec. 5.1 \\
\hline Signals model & $n$ & Regular, $q$ bounded tail & & $\tilde{O}\left(n^{2} q(\epsilon)^{-1} \epsilon^{-4}\right)$ (Sec. 5.2 \\
\hline
\end{tabular}

Table 1: Main results

THEOREM 2. Let $V$ be an arbitrary set, $f$ be an arbitrary function from $V^{n}$ to $[0,1]$, and $X_{1}, \ldots, X_{n} \in V$ be random variables independently drawn from $D^{1}, \ldots, D^{n}$ respectively. Suppose $p=\mathbb{E}_{\mathbf{x} \in \mathbf{D}}[f(\mathbf{x})]$. Let $x_{i 1}, \ldots, x_{i m}$ be $m$ i.i.d. samples from $D^{i}, E^{i}$ be the uniform distribution over $\left\{x_{i 1}, \ldots, x_{i m}\right\}$ for all $i \in[n]$, and $\mathbf{E}=\Pi_{i} E^{i}$. Then, we have

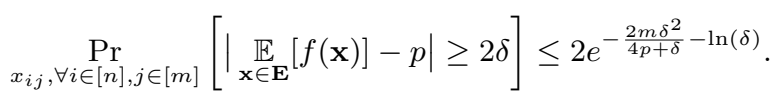

\section{REVENUE MONOTONICITY AND CONCENTRATION INEQUALITY}

In this section prove Theorems 1 and 2

Let $\mathcal{M}$ be the set of all DSIC and IR mechanisms for $n$ buyers and one item, with values in the support of $\mathbf{D}_{0}$. Let $\mathfrak{M}$ be the convex hull of $\mathcal{M}$. Let $\mathcal{D}$ be the set of all product distrubutions $\mathbf{D}$, such that $\mathbf{D} \succeq \mathbf{D}_{0}$, and $\mathfrak{D}$ the convex hull of $\mathcal{D}$. Note that $\mathfrak{D}$ is not the set of all correlated distributions that marginals-wise first order stochastic dominate $\mathbf{D}_{0}$. The theorem would not be true w.r.t. this set 6 We first show the following:

Claim 1.

$$
\max _{M \in \mathfrak{M}} \min _{\mathbf{D} \in \mathfrak{D}} \operatorname{REV}(M, \mathbf{D})=\min _{\mathbf{D} \in \mathfrak{D}} \max _{M \in \mathfrak{M}} \operatorname{REV}(M, \mathbf{D}) .
$$

Proof. We'll use the following theorem, known as Sion's minimax theorem $([19)$ :

Theorem 3 (Sion's minimax TheOrem). Let $X$ be a compact convex subset of a linear topological space and $Y$ a convex subset of a linear topological space. If $f$ is a realvalued function on $X \times Y$ with

- $f(x, \cdot)$ upper semicontinuous and quasi-concave on $Y$, $\forall x \in X$, and

- $f(\cdot, y)$ lower semicontinuous and quasi-convex on $X$, $\forall y \in Y$

then, $\min _{x \in X} \sup _{y \in Y} f(x, y)=\sup _{y \in Y} \min _{x \in X} f(x, y)$.

$\mathfrak{M}$ and $\mathfrak{D}$ are both convex and compact, and $\operatorname{REV}(\cdot, D)$, $\operatorname{Rev}(M, \cdot)$ are both linear functions and continuous by definition.

Let $g(\mathbf{D})=\max _{M \in \mathfrak{M}} \operatorname{REV}(M, \mathbf{D})$. Using the minimax theorem, we show that it is sufficient to prove monotonicity of $g$ in the domain $\mathfrak{D}$, as formalized in this claim.

${ }^{6}$ This is easy to see. Consider two bidders that have either a high or a low value with equal probability, independently. If we make the distributions correlated so that either they both have the high value or low value simultaneously, then the optimum revenue decreases.
Claim 2. $\mathbf{D}_{0} \in \operatorname{argmin}_{\mathbf{D} \in \mathfrak{D}} g(\mathbf{D})$.

Before we prove Claim 2 we'll need the following proposition:

Proposition 1. For all product distributions $\mathbf{D} \in \mathcal{D}$ it holds that:

$$
\nabla_{\mathbf{D}-\mathbf{D}_{0}} g\left(\mathbf{D}_{0}\right) \geq 0
$$

Proof. The proposition is equivalent to the following statement: the revenue of an optimal auction OpT $(\hat{\mathbf{D}})$ for a product distribution $\hat{\mathbf{D}}$ is greater than the revenue of an optimal auction OpT (D) for a product distribution $\mathbf{D}$, if $\hat{\mathbf{D}} \succeq \mathbf{D}$. We'll prove this by constructing a mechanism for $\hat{\mathbf{D}}$ with revenue at least Opt $(\mathbf{D})$.

Let $M$ be the optimal mechanism for $\mathbf{D}$. Let $x_{i}$ and $p_{i}$ be the allocation and payment functions of agent $i$ in $M$ in quantile space, i.e. they take as input a vector of quantiles; $x_{i}$ outputs 0 or 1 , while $p_{i}$ outputs a real number in $[0,1]$, the payment in quantile space. One can go from reported values to quantiles by applying $F_{i}$ to the $i$-th reported value, and vice versa for the payments.

Now, consider the following mechanism for $\hat{\mathbf{D}}$ : translate reported values to quantiles using $\hat{F}_{i}$, run $M$, and map payments to value space using $\hat{F}^{-1}$. This mechanism is truthful, and it yields revenue at least Opт $(\mathbf{D})$ : the distribution over quantiles is uniform, and for a fixed quantile profile the winner is the same, and the payment (in value space) can only be higher: each $\hat{F}_{i}$ is pointwise smaller than $F_{i}$, thus $\hat{F}_{i}^{-1}$ is pointwise bigger than $F_{i}^{-1}$. Since the revenue of this mechanism for $\hat{\mathbf{D}}$ is at least OPT $(\mathbf{D})$, we have that $\operatorname{Opt}(\hat{\mathbf{D}}) \geq \operatorname{Opt}(\mathbf{D})$, which completes the proof.

Now we're ready to prove Claim 2 .

Proof of Claim 2. We'll treat a distribution $\mathbf{D} \in \mathfrak{D}$ as a point in $\mathbb{R}^{c}$, where $c$ is the number of possible valuation tuples, i.e. the size of the support of $\mathfrak{D}$. Since we're only considering discrete distributions, this is a finite dimensional space.

Notice that $g$ is a convex function, since it's the max of linear functions. We'll show that the directional derivate of $g$ along $\mathbf{D}-\mathbf{D}_{0}$ is positive, for all $\mathbf{D} \in \mathfrak{D}$, which implies the claim.

Since $\mathbf{D}$ is in the convex hull of $\mathcal{D}$, it can be written as $\sum_{j} \alpha_{j} \mathbf{D}^{j}$, where $\mathbf{D}^{j} \in \mathcal{D}$, and $\sum_{j} \alpha_{j}=1$. We want to show that $\nabla_{\mathbf{D}-\mathbf{D}_{0}} g\left(\mathbf{D}_{0}\right) \geq 0$. This is equivalent to showing that $\sum_{j} \alpha_{j}\left(\nabla_{\mathbf{D}^{j}-\mathbf{D}_{0}} g\left(\mathbf{D}_{0}\right)\right) \geq 0$, which is implied by Proposition 1 .

The two claims above suffice to get revenue monotonicity as follows. Let $h(M)=\min _{\mathbf{D} \in \mathfrak{D}} \operatorname{REV}(M, \mathbf{D})$, and let 
$M^{*} \in \arg \max _{M} h(M)$. The following sequence of inequalities shows that $M^{*}$ is also an optimal mechanism for $\mathbf{D}_{0}$.

$$
\begin{aligned}
\operatorname{Rev}\left(M^{*}, \mathbf{D}_{0}\right) & \geq \min _{\mathbf{D} \in \mathfrak{D}} \operatorname{Rev}\left(M^{*}, \mathbf{D}\right)=h\left(M^{*}\right)=g\left(\mathbf{D}_{0}\right) \\
& =\max _{M \in \mathfrak{M}} \operatorname{Rev}\left(M, \mathbf{D}_{0}\right) \geq \operatorname{REv}\left(M^{*}, \mathbf{D}_{0}\right) .
\end{aligned}
$$

The second equality above is by Claim 1 and Claim 2 The rest are by definition. Now revenue monotonicity already follows from the above, since it is equivalent to

$$
\operatorname{REV}\left(M^{*}, \mathbf{D}_{0}\right)=\min _{\mathbf{D} \in \mathfrak{D}} \operatorname{ReV}\left(M^{*}, \mathbf{D}\right) .
$$

\subsection{Proof of Theorem 2}

The following lemma follows by Bernstein's inequality.

Lemma 1. Let $\mathbf{y}_{1}, \ldots, \mathbf{y}_{m}$ be $m$ i.i.d. samples from $\mathbf{D}$. Then, we have

$$
\operatorname{Pr}\left[\left|\frac{1}{m} \sum_{j=1}^{m} f\left(\mathbf{y}_{j}\right)-p\right| \geq \delta\right] \leq 2 e^{-\frac{2 m \delta^{2}}{4 p+\delta}} .
$$

We relate the random variables in Theorem 2 and Lemma 1 as follows. First, draw $x_{i 1}, \ldots, x_{i m}$ i.i.d. from $D^{i}$ for all $i \in[n]$, and draw $n$ permutations $\pi_{1}, \ldots, \pi_{n}$ of $[m]$ independently and uniformly at random. Then, let $\mathbf{y}_{j}(\mathbf{x}, \pi)$ be $\left(x_{1 \pi_{1}(j)}, x_{2 \pi_{2}(j)}, \ldots, x_{n \pi_{n}(j)}\right)$ for all $j \in[m]$. Clearly, $\mathbf{y}_{j}(\mathbf{x}, \pi)$ are i.i.d. samples from $\mathbf{E}$. By Lemma 1 .

$$
\operatorname{Pr}_{\mathbf{x}, \pi}\left[\left|\frac{1}{m} \sum_{j=1}^{m} f\left(\mathbf{y}_{j}(\mathbf{x}, \pi)\right)-p\right| \geq \delta\right] \leq 2 e^{-\frac{2 m \delta^{2}}{4 p+\delta}}
$$

Equivalently,

$$
\mathbb{E}_{\mathbf{x}}\left[\operatorname{Pr}_{\pi}\left[\left|\frac{1}{m} \sum_{j=1}^{m} f\left(\mathbf{y}_{j}(\mathbf{x}, \pi)\right)-p\right| \geq \delta\right]\right] \leq 2 e^{-\frac{2 m \delta^{2}}{4 p+\delta}} .
$$

Thus, by Markov's inequality,

$$
\begin{array}{r}
\operatorname{Pr}_{\mathbf{x}}\left[\operatorname{Pr}_{\pi}\left[\left|\frac{1}{m} \sum_{j=1}^{m} f\left(\mathbf{y}_{j}(\mathbf{x}, \pi)\right)-p\right| \geq \delta\right]>\delta\right] \\
\leq 2 e^{-\frac{2 m \delta^{2}}{4 p+\delta}-\ln (\delta)}
\end{array}
$$

Note that $\mathbb{E}_{\mathbf{x} \in \mathbf{E}}[f(\mathbf{x})]=\mathbb{E}_{\pi} \frac{1}{m} \sum_{j=1}^{m} f\left(\mathbf{y}_{j}(\mathbf{x}, \pi)\right)$. So we have

$$
\begin{aligned}
\left|\mathbb{E}_{\mathbf{x} \in \mathbf{E}}[f(\mathbf{x})]-p\right| & \leq \mathbb{E}_{\pi}\left[\frac{1}{m} \sum_{j=1}^{m}\left|f\left(\mathbf{y}_{j}(\mathbf{x}, \pi)\right)-p\right|\right] \\
& \leq \operatorname{Pr}_{\pi}\left[\left|\frac{1}{m} \sum_{j=1}^{m} f\left(\mathbf{y}_{j}(\mathbf{x}, \pi)\right)-p\right| \geq \delta\right] \\
& +\left(1-\operatorname{Pr}_{\pi}\left[\left|\frac{1}{m} \sum_{j=1}^{m} f\left(\mathbf{y}_{j}(\mathbf{x}, \pi)\right)-p\right| \geq \delta\right]\right) \delta \\
& \leq \operatorname{Pr}_{\pi}\left[\left|\frac{1}{m} \sum_{j=1}^{m} f\left(\mathbf{y}_{j}(\mathbf{x}, \pi)\right)-p\right| \geq \delta\right]+\delta
\end{aligned}
$$

Therefore, $\left|\mathbb{E}_{\mathbf{x} \in \mathbf{E}}[f(\mathbf{x})]-p\right| \geq 2 \delta$ implies

$$
\underset{\pi}{\operatorname{Pr}}\left[\left|\frac{1}{m} \sum_{j=1}^{m} f\left(\mathbf{y}_{j}(\mathbf{x}, \pi)\right)-p\right| \geq \delta\right] \geq \delta .
$$

Putting together with (1), the theorem follows.

\section{AUCTIONS WITHOUT SIGNALS}

In this section, we show an $O\left(\frac{n}{\epsilon^{4}} \log \frac{n}{\epsilon}\right)$ upper bound for the non-iid model. One of the difficulties in this model is that the support of the distribution could be unbounded. The main technical ingredient that overcomes this difficulty is to show that we can truncate the values down to a high enough finite quantity without losing too much revenue. This is captured in the following lemma ( Proof in Sec. 4.1).
Lemma 2. Suppose $\frac{1}{4} \geq \epsilon>0$ is a constant. Suppose $\bar{v} \geq \frac{1}{\epsilon} \operatorname{Opt}(\mathbf{D})$. Let $\bar{D}^{1}, \ldots, \bar{D}^{n}$ be the distributions obtained by truncating $D^{1}, \ldots, D^{n}$ at $\bar{v}$, i.e., a sample $\bar{v}_{i}$ from $\bar{D}^{i}$ is obtained by first sampling $v_{i}$ from $D^{i}$ and then letting $\bar{v}_{i}=\min \left\{v_{i}, \bar{v}\right\}$. Then, we have

$$
\operatorname{Opt}\left(\Pi_{i} \bar{D}^{i}\right) \geq(1-4 \epsilon) \operatorname{Opt}(\mathbf{D})
$$

In order to use Lemma 2, we need to estimate the optimal revenue up to a constant factor. To do this, we employ a bootstrapping approach. We consider families of simple mechanisms, namely, selling to each buyer separately and the VCG mechanism with duplicates 7 These simple mechanisms require relatively few samples to implement and get a good approximation of the optimal revenue of the original problem (e.g., 11]). We run these simpler mechanisms with $\Theta\left(n \epsilon^{-1} \log \frac{n}{\epsilon}\right)$ samples to find a constant approximation of optimal revenue using Algorithm 11. whose role is summarized in Lemma 3

LEMMA 3. With probability $1-O(\epsilon)$, we have

$$
\frac{1}{8} \mathrm{OPT}(\mathbf{D}) \leq \operatorname{Apx} \leq 8 \mathrm{OPT}(\mathbf{D}) .
$$

We'll first prove the following straightforward Lemma:

Lemma 4. With probability $1-O(\epsilon)$, we have

$$
\text { Opt }(\mathbf{D}) \leq \mathrm{SREV} \leq 2 n \mathrm{OPT}(\mathbf{D})
$$

Proof. $\max _{i \in[n]}$ Opt $\left(D^{i}\right) \leq$ Opt $(\mathbf{D}) \leq \sum_{i=1}^{n}$ Opt $\left(D^{i}\right)$. Also, $\mathrm{SREV}_{i}$ is a 2 approximation of OPT $\left(D^{i}\right)$. The lemma follows.

Proof of Lemma 3. By Lemma 2 and Lemma 4, the optimal revenue when the values are truncated at $\frac{1}{\epsilon} \mathrm{SREV} \geq$ $\frac{1}{\epsilon} \mathrm{Opt}(\mathbf{D})$, denoted as $\overline{\mathrm{OPT}}$, is at least $(1-O(\epsilon)) \operatorname{Opt}(\mathbf{D})$. On one hand, the truncated distributions are also regular. So the expected revenue of running VCG with duplicate w.r.t. the truncated values, denoted as $\overline{\mathrm{VCG}}$, is a 2approximation of the optimal revenue of the truncated distributions (Theorem 4.4 of 11$]$ ), i.e., $\overline{\mathrm{VCG}} \geq \frac{1}{2} \overline{\mathrm{OPT}}$. On the other hand, we have $\overline{\mathrm{VCG}} \leq 2 \overline{\mathrm{OPT}}$ since we are running a mechanism with two copies of each buyer. Further, the revenue from each run of the VCG with duplicate is at most $\frac{1}{\epsilon} \mathrm{SREV} \leq \frac{2 n}{\epsilon} \mathrm{OPT}(\mathbf{D})$ because the values are truncated at $\frac{1}{\epsilon}$ SREv. Putting together, by Bernstein inequality, with probability $1-O(\epsilon)$, the average revenue from the $m_{1}=\Theta\left(n \epsilon^{-1} \log \epsilon^{-1}\right)$ runs of VCG with duplicates are upper and lower bounded as follows:

$$
\begin{aligned}
& \mathrm{Apx} \leq 2 \overline{\mathrm{VCG}} \leq 4 \overline{\mathrm{OPT}} \leq 8 \mathrm{OPT}(\mathbf{D}) \\
& \mathrm{Apx} \geq \frac{1}{2} \overline{\mathrm{VCG}} \geq \frac{1}{4} \overline{\mathrm{OPT}} \geq \frac{1}{8} \mathrm{OPT}(\mathbf{D})
\end{aligned}
$$

We further show that discretizing the value space into powers of $1-\epsilon$ doesn't hurt the revenue too much:

Lemma 5. Given any product value distribution $\mathbf{D}^{\prime}$, and $\mathbf{D}^{\prime \prime}$ obtained by rounding the values from $\mathbf{D}^{\prime}$ to the closest power of $1-\epsilon$, we have Орт $\left(\mathbf{D}^{\prime \prime}\right) \geq(1-\epsilon)$ Opt $\left(\mathbf{D}^{\prime}\right)$.

${ }^{7}$ Another potential approach is to use VCG with monopoly reserves, which also gives similar guarantees. 

1: For all $i \in[n]$, use $O\left(\log \frac{n}{\epsilon}\right)$ samples to compute a 2-approximation of the optimal revenue when buyer $i$ was the only buyer, denoted as $\mathrm{SREV}_{i}$, i.e., $\operatorname{Opt}\left(D^{i}\right) \leq \operatorname{SREv}_{i} \leq 2 \mathrm{Opt}\left(D^{i}\right)$ (e.g., 12 ).
2: Let $\mathrm{SREV}=\sum_{i=1}^{n} \mathrm{SREV}_{i}$.
3: Let $m_{1}=\Theta\left(n \epsilon^{-1} \log \epsilon^{-1}\right)$ and let there be $2 m_{1}$ fresh samples.
4: Run VCG with two copies of each buyer $m_{1}$ times, capping the values by $\frac{1}{\epsilon} \mathrm{SREV}$.
5: Output the average revenue, denoted as APX.

Algorithm 1: Computing an $\mathrm{O}(1)$ approximation of the optimal revenue

Proof. Let $M^{\prime}$ be the optimal mechanism with respect to $\mathbf{D}^{\prime}$. Consider a mechanism $M^{\prime \prime}$ with the following allocation rule: (1) let $q_{i}^{\prime \prime}$ 's be the quantiles of $v_{i}^{\prime \prime}$ 's respectively w.r.t. $\mathbf{D}^{\prime \prime} ;(2)$ let $v_{i}^{\prime}$ 's be the values that correspond to $q_{i}^{\prime \prime}$ 's w.r.t. $\mathbf{D}^{\prime}$; (3) use the allocation of mechanism $M^{\prime \prime}$ for values $v_{i}^{\prime}$ 's. Clearly, the above allocation rule is monotone. Thus, there is a payment rule that makes it truthful.

Next, we analyze the revenue of $M^{\prime \prime}$. Given any quantiles $\mathbf{q} \in[0,1]^{n}$, let $\mathbf{v}^{\prime}$ and $\mathbf{v}^{\prime \prime}$ be the corresponding values w.r.t. $\mathbf{D}^{\prime}$ and $\mathbf{D}^{\prime \prime}$ respectively. We have that the allocation of $M^{\prime}$ for $\mathbf{v}^{\prime}$ is the same as the allocation of $M^{\prime \prime}$ for $\mathbf{v}^{\prime \prime}$, i.e., the set of winners in both cases are the same. Further, the payment is equal to the threshold value of each winner above which he remains a winner; the quantiles of the threshold values in both cases are the same. Recall that $\mathbf{D}^{\prime \prime}$ is obtained by rounding the values from $\mathbf{D}^{\prime}$ to the closest power of $1-\epsilon$. So for any given quantile, the corresponding values in $\mathbf{D}^{\prime}$ and $\mathbf{D}^{\prime \prime}$ differs by at most a $1-\epsilon$ factor. Thus, the revenue of $M^{\prime \prime}$ for values $\mathbf{v}^{\prime \prime}$ is at least a $1-\epsilon$ fraction of that of $M^{\prime}$ for value $\mathbf{v}^{\prime}$. Since this holds for all quantiles $\mathbf{q}$, the lemma follows.

The final algorithm we use is: find an approximation to Opt (D), discretize the values accordingly, and run the Myerson auction on the samples, rounded to the discrete set. This is summarized in Algorithm 2

1: Compute Apx using Algorithm 1

2: Let $V=\left\{\frac{8}{\epsilon} \operatorname{Apx}, \frac{8(1-\epsilon)}{\epsilon} \operatorname{ApX}, \frac{8(1-\epsilon)^{2}}{\epsilon} \operatorname{Apx}, \ldots, \frac{\epsilon}{8 n} \mathrm{APX}, 0\right\}$.

3: Let there be $m_{2}=\Theta\left(n \epsilon^{-4} \log ^{2} \frac{n}{\epsilon}\right)$ fresh samples $\mathbf{v}_{1}, \ldots, \mathbf{v}_{m_{2}}$.

4: Round all coordinates of each $\mathbf{v}_{i}$ down to the closest value in $V$;

let $\mathbf{v}_{1}^{\prime}, \ldots, \mathbf{v}_{m_{2}}^{\prime}$ denote the rounded value vectors.

5: Let $E^{i}$ denote the empirical distribution of buyer $i$ , i.e., the uniform distribution over $v_{1 i}, v_{2 i}, \ldots, v_{m_{2} i}$. 6: Run Myerson auction w.r.t. $\mathbf{E}=E^{1} \times \cdots \times E^{n}$.

Algorithm 2: Empirical Myerson with Preconditioning

\section{TheOrem 4. Algorithm 2 is a $1-O(\epsilon)$ approximation.}

Proof. For any product distribution over $V^{n}$, Myerson's optimal auction picks the bidder with the largest ironed virtual surplus, breaking ties uniformly at random. Equivalently, there is a mapping $\sigma:[n] \times V \mapsto\{1,2, \ldots, n|V|\}$ such that given any value profile $v_{1}, \ldots, v_{n} \in V$, the Myerson auction allocates the item to $i^{*}=\arg \min _{i \in[n]} \sigma\left(i, v_{i}\right)$. Let $M_{\sigma}$ denote the auction corresponding to a mapping $\sigma$. Therefore, there are at most $(n|V|)^{n|V|}$ different Myerson auctions for product distributions over $V^{n}$.

For any given $\sigma$, we apply Theorem 2 with $\mathbf{D}$ being the distribution of rounded values $\overline{\mathbf{D}}$, with $f(\mathbf{v})$ being the revenue of $M_{\sigma}$ when the values are $\mathbf{v}$ (divided by $\frac{8}{\epsilon}$ APX so that $f(\mathbf{v}) \in[0,1])$, and with $m=m_{2}=\Theta\left(n \epsilon^{-4} \log ^{2} \frac{n}{\epsilon}\right)$ samples (for a sufficiently large constant inside the big- $\Theta$ notation). Note that $p=\mathbb{E}_{\mathbf{x} \in \mathbf{D}}[f(\mathbf{x})] \leq \operatorname{Opt}(\overline{\mathbf{D}}) /\left(\frac{8}{\epsilon} \mathrm{APX}\right)=O(\epsilon)$, and that $\delta=(\epsilon \operatorname{Opt}(\overline{\mathbf{D}})) /\left(\frac{8}{\epsilon} \operatorname{Apx}\right)=\Theta\left(\epsilon^{2}\right)$. We get the following (where the probability is taken over the randomness in the $m_{2}$ samples):

$$
\begin{aligned}
& \operatorname{Pr}\left[\left|\operatorname{Rev}\left(M_{\sigma}, \mathbf{E}\right)-\operatorname{Rev}\left(M_{\sigma}, \overline{\mathbf{D}}\right)\right| \geq 2 \epsilon \operatorname{OPT}(\overline{\mathbf{D}})\right] \\
& \leq 2 e^{-\frac{2 m \delta^{2}}{4 p+\delta}-\ln (\delta)}=2 e^{-\Omega\left(\frac{n}{\epsilon} \log ^{2} \frac{n}{\epsilon}\right)} \leq \epsilon(n|V|)^{-n|V|}
\end{aligned}
$$

where the last inequality use the fact that $|V|=O\left(\epsilon^{-1} \log \frac{n}{\epsilon}\right)$. By union bound

$$
\left|\operatorname{Rev}\left(M_{\sigma}, \mathbf{E}\right)-\operatorname{Rev}\left(M_{\sigma}, \overline{\mathbf{D}}\right)\right|<2 \epsilon \operatorname{Rev}\left(M_{\sigma}, \overline{\mathbf{D}}\right),
$$

for all $\sigma$ with probability at least $1-\epsilon$. Since Algorithm 2 uses the optimal auction w.r.t. $\mathbf{E}$, it gets at least (1 $2 \epsilon) \operatorname{Opt}(\overline{\mathbf{D}})$ revenue. Putting together with Lemma 2 and Lemma 5 proves the theorem.

\subsection{Proof of Lemma 2}

Note that when $n=1$, the lemma follows easily by concavity of the revenue curves of regular distributions. What is the extra challenge with multiple buyers? If the buyers' values are all below the truncation point $\bar{v}$, the virtual values are the same in both cases and, thus, the revenue is the same. If exactly one of the buyers' value is larger than $\bar{v}$, the analysis is similar to the $n=1$ case. It remains to bound the revenue when more than one buyers' values are larger than $\bar{v}$, in which case the competition among the buyers may give an extra edge to the untruncated case. It turns out that truncations are so rare such that the revenue from the third case of the untruncated setting can be charged to the revenue from the second case of the truncated setting.

Truncations are Rare. Let $\bar{q}_{i}=1-F(\bar{v})$ denote the quantile of value $\bar{v}$ w.r.t. $D^{i}$. Let $\phi_{i}\left(q_{i}\right)$ and $\bar{\phi}_{i}\left(q_{i}\right)$ denote the virtual value at quantile $q_{i}$ w.r.t. $D^{i}$ and $\bar{D}^{i}$ respectively. Then, $\bar{\phi}_{i}\left(q_{i}\right)=\phi_{i}\left(q_{i}\right)$ if $\bar{q}_{i}<q_{i} \leq 1$ and $\bar{v}$ otherwise. It is easy to see that for any $i \in[n]$, we have $\bar{q}_{i} \leq \epsilon$. We also show the following more refined bound on $\bar{q}_{i}$ s.

Lemma 6. The probability that at least one buyer has valuation greater than or equal to $\bar{v}$ satisfies

$$
\epsilon \geq 1-\prod_{i \in[n]}\left(1-\bar{q}_{i}\right) \geq(1-\epsilon) \sum_{i \in[n]} \bar{q}_{i} .
$$

Charging Argument. Note that both $D^{i}$ and $\bar{D}^{i}$ are regular, so Myerson's auction simply picks the buyer with the maximum virtual surplus, and the revenue is the virtual welfare thus obtained. Given a quantile vector $\mathbf{q}$, let $H(\mathbf{q})=$ $\left\{i \in[n]: q_{i} \leq \bar{q}_{i}\right\}$ denote the buyers whose values are higher than $\bar{v}$; let $L(\mathbf{q})=[n] \backslash H(\mathbf{q})$ denote the set of buyers whose values are smaller than $\bar{v}$. We bound the ratio separately, 
for the high value buyers and the low value buyers, as below.

$$
\begin{aligned}
\int_{[0,1]^{n} \backslash \prod_{j}\left[\bar{q}_{j}, 1\right]} \max _{i} \bar{\phi}_{i}\left(q_{i}\right) d^{n} \mathbf{q} \\
\geq(1-2 \epsilon) \int_{[0,1]^{n}} \max _{i \in H(\mathbf{q})} \phi_{i}\left(q_{i}\right) d^{n} \mathbf{q}, \text { and } \\
\int_{\prod_{j}\left[\bar{q}_{j}, 1\right]} \max _{i} \bar{\phi}_{i}\left(q_{i}\right) d^{n} \mathbf{q} \\
\geq(1-2 \epsilon) \int_{[0,1]^{n}} \max _{i \in L(\mathbf{q})} \phi_{i}\left(q_{i}\right) d^{n} \mathbf{q} .
\end{aligned}
$$

Before we prove Equations 2 and 3 we need the following Lemma:

LEMma 7. For any $i \in[n]$, we have $\bar{q}_{i} \leq \epsilon$.

Proof. Note that serving buyer $i$ as if he was the only buyer with a reserve price $\bar{v}$ give revenue $\bar{q}_{i} \bar{v}$, which must be less than or equal to the optimal revenue Opt $(\mathbf{D})$. The lemma then follows from that $\bar{v} \geq \frac{1}{\epsilon} \mathrm{OPT}(\mathbf{D})$.

\section{Proof of (2):}

Note that for any $i$ and any $q_{i}<\bar{q}_{i}, \bar{\phi}_{i}\left(q_{i}\right)=\bar{v}$. Thus, the LHS of 2 is lower bounded by

$$
\int_{[0,1]^{n} \backslash \prod_{i}\left[\bar{q}_{i}, 1\right]} \bar{v} d^{n} \mathbf{q}=\left(1-\prod_{i \in[n]}\left(1-\bar{q}_{i}\right)\right) \bar{v} \geq(1-\epsilon) \sum_{i \in[n]} \bar{q}_{i} \bar{v}
$$

where the inequality follows by Lemma 6 On the other hand, the RHS (omitting the $1-2 \epsilon$ factor) is upper bounded by

$$
\begin{aligned}
\int_{[0,1]^{n}} \max _{i \in H(\mathbf{q})} \phi_{i}\left(q_{i}\right) d^{n} \mathbf{q} & \leq \int_{[0,1]^{n}} \sum_{i \in H(\mathbf{q})} \max \left\{\phi_{i}\left(q_{i}\right), 0\right\} d^{n} \mathbf{q} \\
& \leq \sum_{i \in[n]} \int_{\left[0, \bar{q}_{i}\right]} \max \left\{\phi_{i}\left(q_{i}\right), 0\right\} d q_{i}
\end{aligned}
$$

Therefore, to show (2), it suffices to show that for every $i \in[n]$,

$$
\bar{q}_{i} \bar{v} \geq(1-\epsilon) \int_{q_{i} \in\left[0, \bar{q}_{i}\right]} \max \left\{\phi_{i}\left(q_{i}\right), 0\right\}
$$

Let $R_{i}(q)$ denote the revenue of a single-buyer auction with a reserve price that has quantile $q$ w.r.t. $D_{i}$. The LHS of the above is the revenue of a single-buyer auction with reserve price $\bar{v}$ w.r.t. $D_{i}$, i.e., $R_{i}\left(\bar{q}_{i}\right)$. The RHS is the maximum revenue of a single-buyer auction with a reserve price at least $\bar{v}$, i.e., $\max _{q \in\left[0, \bar{q}_{i}\right]} R_{i}(q)$. Note that $D_{i}$ 's being regular implies that $R_{i}(q)$ is a concave function over $[0,1]$ and $R_{i}(1) \geq 0$. Thus, we have that $R_{i}\left(\bar{q}_{i}\right) \geq\left(1-\bar{q}_{i}\right) \max _{q \in\left[0, \bar{q}_{i}\right]} R_{i}(q)$. The inequality then follows by $\bar{q}_{i} \leq \epsilon$ (Lemma 7 ).

\section{Proof of $(3)$ :}

Note that for any $\mathbf{q} \in \prod_{j \in[n]}\left[\bar{q}_{j}, 1\right]$, we have $L(\mathbf{q})=[n]$. So we can rewrite the LHS of (3) as follows.

$$
\int_{\prod_{j}\left[\bar{q}_{j}, 1\right]} \max _{i \in[n]} \phi_{i}\left(q_{i}\right) d^{n} \mathbf{q}=\int_{\prod_{j}\left[\bar{q}_{j}, 1\right]} \max _{i \in L(\mathbf{q})} \phi_{i}\left(q_{i}\right) d^{n} \mathbf{q}
$$

For any $\mathbf{q}_{-i} \in[0,1]^{n-1}$, any $q_{i}<\bar{q}_{i} \leq q_{i}^{\prime}$, consider $\mathbf{q}=$ $\left(q_{i}, \mathbf{q}_{-i}\right)$ and $\mathbf{q}^{\prime}=\left(q_{i}^{\prime}, \mathbf{q}_{-i}\right)$. We have that $L(\mathbf{q}) \subset L\left(\mathbf{q}^{\prime}\right)$, and for any $i \in L(\mathbf{q}), q_{i}=q_{i}^{\prime}$. Thus, $\max _{i \in L(\mathbf{q})} \phi_{i}\left(q_{i}\right) \leq$ $\max _{i \in L\left(\mathbf{q}^{\prime}\right)} \phi_{i}\left(q_{i}^{\prime}\right)$. Therefore, we have that

$$
\begin{aligned}
& \int_{\prod_{j}\left[\bar{q}_{j}, 1\right]} \max _{i \in L(\mathbf{q})} \phi_{i}\left(q_{i}\right) d^{n} \mathbf{q} \\
& \geq\left(1-\bar{q}_{1}\right) \int_{[0,1] \times \prod_{j=2}^{n}\left[\bar{q}_{j}, 1\right]} \max _{i \in L(\mathbf{q})} \phi_{i}\left(q_{i}\right) d^{n} \mathbf{q} \\
& \geq\left(1-\bar{q}_{1}\right)\left(1-\bar{q}_{2}\right) \int_{[0,1]^{2} \times \prod_{j=3}^{n}\left[\bar{q}_{j}, 1\right]} \max _{i \in L(\mathbf{q})} \phi_{i}\left(q_{i}\right) d^{n} \mathbf{q} \\
& \geq \ldots \\
& \geq \prod_{j \in[n]}\left(1-\bar{q}_{j}\right) \int_{[0,1]^{n}} \max _{i \in L(\mathbf{q})} \phi_{i}\left(q_{i}\right) d^{n} \mathbf{q}
\end{aligned}
$$

Finally, by Lemma 6 , we have that $\prod_{j \in[n]}\left(1-\bar{q}_{j}\right) \geq 1-\epsilon$. Putting together proves (3).

\section{SIGNAL AUCTIONS UPPER BOUNDS}

\subsection{Single Agent}

In this subsection we sketch a proof of the $O\left(\frac{\log (1 / \epsilon)}{q(\epsilon) \epsilon^{3}}\right)$ upper bound for the Signals model, for $n=1$. Our auction is simply a posted price. The main challenge is the scarcity of samples with the same signal as the buyer (in fact, there might be none). The idea is to find an auxiliary distribution such that (1) we have enough samples to find a nearly optimal price for it, and (2) it is stochastically dominated by the buyer's prior. Then, we can lower bound the revenue of the auction with the optimal revenue of the auxiliary distribution. To this end, we consider a number of signals in the sample that are just below the signal of the bidder, and use the mixture of the corresponding distributions as our auxiliary distribution. The price posted is the best price for the empirical distribution of values corresponding to these signals, guarded against using too few of these values. The algorithm is summarized in Algorithm 3

1: Samples: $\left(\hat{v}_{1}, \hat{\sigma}_{1}\right),\left(\hat{v}_{2}, \hat{\sigma}_{2}\right), \ldots,\left(\hat{v}_{m}, \hat{\sigma}_{m}\right)$, where $\hat{\sigma}_{1} \geq \hat{\sigma}_{2} \geq \cdots \geq \hat{\sigma}_{m}$.

2: Parameter: $\epsilon$

3: Input: Signal $\sigma$ (the value $v$ is unobserved); suppose $\hat{\sigma}_{k} \geq \sigma \geq \hat{\sigma}_{k+1}$.

4: Auction: Let $c=\Theta\left(\epsilon^{-3} \log \epsilon^{-1}\right)$, and $\ell=\min \{c, m-k\}$.

Post the $\epsilon$-guarded empirical reserve price with respect to $v_{k+1}, v_{k+2}, \ldots, v_{k+\ell}$, defined as:

$p_{\text {emp }} \stackrel{\text { def }}{=} \arg \max _{\hat{v}_{k+j}: \ell \leq j \leq \ell} \hat{v}_{k+j} \cdot\left|\left\{\hat{v}_{k+i}: \hat{v}_{k+i} \geq \hat{v}_{k+j}, 1 \leq i \leq \ell\right\}\right|$

Algorithm 3: Computing a $1-\Theta(\epsilon)$ approximation of the optimal revenue

In the analysis, we divide the realization of all the random variables, $\left(\hat{v}_{j}, \hat{\sigma}_{j}\right)$ for $j \in[m]$, and the eventual realization $(v, \sigma)$ of the bidder, into 3 stages as follows.

1. Sample $m+1$ signals $\sigma_{1}^{\prime}, \sigma_{2}^{\prime}, \ldots, \sigma_{m+1}^{\prime}$ i.i.d. from $F_{\mathcal{D}, \sigma}$; suppose $\sigma_{1}^{\prime} \geq \sigma_{2}^{\prime} \geq \cdots \geq \sigma_{m+1}^{\prime}$.

2. Pick $i \in[m+1]$ uniformly at random and let $\sigma=\sigma_{i}^{\prime}$; let $\hat{\sigma}_{1}, \hat{\sigma}_{2}, \ldots, \hat{\sigma}_{m}$ be the other $m$ signals.

3. Sample $m+1$ values, $\hat{v}_{j} \sim D^{\hat{\sigma}_{j}}$ and $v \sim D^{\sigma}$, independently. 
We lower bound the revenue of the auction successively over the randomization in the different stages. Using revenue monotonicity and the stochastic dominance between signals, we lower bound the revenue over the randomness of the last stage (Lemma 9). The lemma is similar to Theorem 3.6 of 12 with the extra complication that the sample values are not iid from the auxiliary distribution. Next, we lower bound the revenue over the randomness of the last two stages (Lemma 10), and finally over the randomness of all three stages (Lemma 11). The revenue guarantee then follows from Definition 1 .

Let $\operatorname{REV}(p, D)$ denote the expected revenue of posting price $p$ when the underlying distribution is $D$.

Definition 2 (Truncated Distributions). Given any distribution $D$ over $[0,+\infty)$ and any $0<\delta<1$, let $D_{\delta}^{\text {trunc }}$ be the distribution obtained by replacing the entire top $\delta$ of the probability mass of $D$ with a point mass. Formally, if $F$ is the c.d.f. of $D$ then the c.d.f. of $D_{\delta}^{\text {trunc }}$ is identical to $F$ for all values less than $F^{-1}(1-\delta)$, and the c.d.f of $D_{\delta}^{\text {trunc }}$ at $F^{-1}(1-\delta)$ is 1 .

Let $\hat{D}$ denote the mixed distribution of $D^{\hat{\sigma}_{k+1}}, \ldots, D^{\hat{\sigma}_{k+\ell}}$. That is, if we want to draw a value from $\hat{D}$, we first sample $i$ from $\{1,2, \ldots, \ell\}$ uniformly at random, and then draw a value from $D^{\hat{\sigma}_{k+i}}$. It is easy to verify the following claims:

Claim 3. $D^{\sigma} \succeq \hat{D}_{\epsilon}^{\text {trunc }} \succeq D^{\hat{\sigma}_{k+\ell}}$ trunc $^{\text {trum }}$.

Claim 4. If $D \succeq D^{\prime}$, then for any price $p \geq 0$ we have $\operatorname{REV}(p, D) \geq \operatorname{Rev}\left(p, D^{\prime}\right)$.

Claim 5. Opt $\left(D_{\epsilon}^{\text {trunc }}\right) \geq(1-\epsilon)$ Opt $(D)$, for all regular distributions $D$.

We first need to show that:

LEMMA 8.

$$
\mathbb{E}\left[\operatorname{REV}\left(p_{\text {emp }}, \hat{D}_{\epsilon}^{\text {trunc }}\right) \mid \hat{\sigma}_{1}, \ldots, \hat{\sigma}_{m}, \sigma\right] \geq(1-\epsilon)^{2} \text { Opt }\left(\hat{D}_{\epsilon}^{\text {trunc }}\right),
$$

where the expectation is over the random draws of values from the $D^{\hat{\sigma}_{i}}$ 's.

Proof. For the purposes of this proof we'll relax notation and not write the conditioning on $\hat{\sigma}_{1}, \ldots, \hat{\sigma}_{m}, \sigma$. Let $p^{*}$ be the optimal price for $\hat{D}_{\epsilon}^{\text {trunc }}$, i.e. $\operatorname{REV}\left(p^{*}, \hat{D}_{\epsilon}^{\text {trunc }}\right)=$ Opt $\left(\hat{D}_{\epsilon}^{\text {trunc }}\right)$. Also let $\hat{E}_{\epsilon}^{\text {trunc }}$ be the uniform distribution over $V=\left\{\hat{v}_{k+j}: \epsilon \ell \leq j \leq \ell\right\}$, where $\hat{v}_{k+j}$ is drawn from $D^{\hat{\sigma}_{k+j}}$. Using standard Chernoff bounds it's easy to see that w.h.p.:

$$
\operatorname{REV}\left(p^{*}, \hat{E}_{\epsilon}^{\text {trunc }}\right) \geq(1-\epsilon) \operatorname{REV}\left(p^{*}, \hat{D}_{\epsilon}^{\text {trunc }}\right),
$$

i.e. expected revenue of $p^{*}$ in $\hat{E}_{\epsilon}^{\text {trunc }}$ is a good estimate of it's revenue in $\hat{D}_{\epsilon}^{\text {trunc }}$. Using a similar proof (plus union bound) we can show that the same is true for all $\hat{v}_{k+z} \in V$, and thus:

$$
\operatorname{REV}\left(p_{\text {emp }}, \hat{D}_{\epsilon}^{\text {trunc }}\right) \geq(1-\epsilon) \operatorname{REV}\left(p_{\text {emp }}, \hat{E}_{\epsilon}^{\text {trunc }}\right),
$$

Finally, we'll show that the value in $V$ with the highest revenue in $\hat{E}_{\epsilon}^{\text {trunc }}$, i.e. $p_{\text {emp }}$, has revenue in $\hat{E}_{\epsilon}^{\text {trunc }}$ at least the revenue of $p^{*}$ in $\hat{E}_{\epsilon}^{\text {trunc }}$ :

$$
\operatorname{REV}\left(p_{\text {emp }}, \hat{E}_{\epsilon}^{\text {trunc }}\right) \geq \operatorname{REV}\left(p^{*}, \hat{E}_{\epsilon}^{\text {trunc }}\right)
$$

$\hat{E}_{\epsilon}^{\text {trunc }}$ is uniform over discrete values; the optimal (in terms of revenue) posted price chosen out of those values is also optimal overall. Putting it all together we get that w.h.p.:

$$
\begin{array}{rlr}
\operatorname{REV}\left(p_{\text {emp }}, \hat{D}_{\epsilon}^{\text {trunc }}\right) & \geq(1-\epsilon) \operatorname{REV}\left(p_{\text {emp }}, \hat{E}_{\epsilon}^{\text {trunc }}\right) & \text { Eqn: } 5 \\
& \geq(1-\epsilon) \operatorname{REV}\left(p^{*}, \hat{E}_{\epsilon}^{\text {trunc }}\right) & \text { Eqn: } 6 \\
& \geq(1-\epsilon)^{2} \operatorname{REV}\left(p^{*}, \hat{D}_{\epsilon}^{\text {trunc }}\right) & \text { Eqn: } 4 \\
& =(1-\epsilon)^{2} \operatorname{OpT}\left(\hat{D}_{\epsilon}^{\text {trunc }}\right) &
\end{array}
$$

The main lemma, whose proof we omit, for this stage is the following:

LEMMA 9.

$\mathbb{E}\left[\operatorname{ReV}\left(p_{e m p}, D^{\sigma}\right) \mid \hat{\sigma}_{1}, \ldots, \hat{\sigma}_{m}, \sigma\right] \geq(1-\epsilon)^{3}$ Opт $\left(D^{\hat{\sigma}_{k+\ell}}\right)$.

Next, we lower bound the revenue of the above auction over the randomness of the last two stages:

LEMMA 10.

$\mathbb{E}\left[\operatorname{REV}\left(p_{e m p}, D^{\sigma}\right) \mid \sigma_{1}^{\prime}, \ldots, \sigma_{m+1}^{\prime}\right] \geq \frac{(1-\epsilon)^{3}}{m+1} \sum_{i=\ell+1}^{m+1}$ OPT $\left(D^{\sigma_{i}^{\prime}}\right)$.

Finally, we will lower bound the revenue of the above auction over the randomness of all three stages.

LEMMA 11.

$\mathbb{E}\left[\operatorname{REV}\left(p_{\text {emp }}, D^{\sigma}\right)\right] \geq(1-\epsilon)^{3} \int_{0}^{F_{\mathcal{D}, \sigma}^{-1}\left(1-\frac{c}{m+1}\right)} \operatorname{Opt}\left(D^{\sigma}\right) d F_{\mathcal{D}, \sigma}$.

Proof. Recall that $\sigma_{1}^{\prime}, \ldots, \sigma_{m+1}^{\prime}$ are i.i.d. from $F_{\mathcal{D}, \sigma}$. Thus, we have

$$
\frac{1}{m+1} \sum_{i=1}^{m+1} \operatorname{OpT}\left(D^{\sigma_{i}^{\prime}}\right)=\int_{0}^{1} \operatorname{OPT}\left(D^{\sigma}\right) d F_{\mathcal{D}, \sigma}
$$

Let us compare the LHS of the above with the RHS of 10 (removing the $(1-\epsilon)^{3}$ factor), i.e., $\frac{1}{m+1} \sum_{i=\ell+1}^{m+1}$ Opt $\left(D^{\sigma_{i}^{\prime}}\right)$. The latter drops $\ell$ of the $m+1$ signals in the summation and, thus, effectively drops $\frac{\ell}{m+1}$ of the probability mass in the expectation. Recall that $D^{\sigma}$ stochastically dominates $D^{\sigma^{\prime}}$ for any $\sigma \geq \sigma^{\prime}$. So the worst-case scenario is dropping the contributions from signals $\sigma \in\left[1-\frac{c}{m+1}, 1\right]$. The lemma then follows.

\subsection{Multiple agents}

In this subsection, we show how to solve the multi-agent problem in the Signals model, building on techniques in the single-agent problem and in the non-iid model of the multiagent problem. Formally, we prove the following sample complexity upper bound.

TheOREM 5 (UPPER BOUND FOR MUlTiple AGENTS). There exists a mechanism for selling to $n$ agents in the Signals model, that has a sample complexity, against regular distributions which have a q-bounded tail in the signal space, of

$$
O\left(\frac{n^{2}}{q(\epsilon) \epsilon^{4}} \log ^{2} \frac{n}{\epsilon}\right)
$$




\section{High-level ideas.}

We combine the ideas from the single-agent signal case (Section 5.1) and the multi-agent no-signal case (Section 4 ). For each agent, consider $\ell=\Theta\left(\frac{n^{2}}{\epsilon^{4}} \log \frac{n}{\epsilon}\right)$ sample signals that are closest to the agent's signal from below, and using the corresponding $\ell$ sample values in place of the actual samples from his true distribution, we run the the empirical Myerson's auction with preconditioning in Section 4. Like in the single agent case, we would like to show that the expected revenue of the auction is almost as good as the optimal revenue when each agent's distribution is replaced by the distribution whose signal is the $\ell$-th closest to the agent's signal from below. Then, we can use the small tail assumption in the signal space to show that the auction gives nearly optimal revenue.

There are two caveats, one in the algorithm and one in the analysis. First, the implementation of the empirical Myerson's auction in Section 4 uses the VCG mechanism with duplicate agents to estimate the optimal revenue up to a constant factor, exploiting regularity of the distributions. The algorithm in the Signals model, however, needs to estimate the optimal revenue when each agent's distribution is replaced by the mixture of the $\ell$ distributions whose signals are closest to the agent's signal from below. The mixture of regular distributions is not necessarily regular. Thus, we turn to a coarser $n$-approximation.

Further, the analysis for the single-agent case uses a strong notion of revenue monotonicity, which holds regardless of the mechanism being used 8 With multiple agents, we need to make do with the weaker notion of revenue monotonicity in Theorem 1, which only holds for the optimal auction for the dominated distribution. This is still not directly applicable because we can only find the optimal auction for the empirical distribution w.r.t. the mixture of the $\ell$ distributions whose signals are closest to the agent's signal from below, which is not necessarily dominated by the true distribution for the agent's signal, due to the randomness in the sample values. To this end, we further introduce a coupling argument to show that the optimal auction for the empirical distribution gives essentially the same revenue guarantee.

\section{Algorithm.}

The algorithm is presented in Algorithm 4 . We can implement line 7 using the approach in, for example, [12, with an extra complication that $\hat{v}_{k_{i}+1}, \ldots, \hat{v}_{k_{i}+\ell}$ are not iid samples from $\bar{D}^{i}$. Note that this extra complication is the same problem that we have in the single-buyer case (Lemma 8) and, thus, can be handled the same way. We will omit the details.

\section{Analysis.}

Similar to the single-buyer case, we divide the realization of all the random variables into 3 stages as follows.

1. Sample $m+n$ signals $\sigma_{1}^{\prime}, \sigma_{2}^{\prime}, \ldots, \sigma_{m+n}^{\prime}$ i.i.d. from $F_{\mathcal{D}, \sigma}$; suppose $\sigma_{1}^{\prime} \geq \sigma_{2}^{\prime} \geq \cdots \geq \sigma_{m+n}^{\prime}$.

2. Pick a subset of $n$ indices $i_{1} \neq \cdots \neq i_{n} \in[m+n]$ uniformly at random and let $\sigma_{j}=\sigma_{i_{j}}^{\prime}$ for all $j \in[n]$; let $\hat{\sigma}_{1} \geq \cdots \geq \hat{\sigma}_{m}$ be the other $m$ signals. (Note that

${ }^{8}$ This is true in the single agent case since these mechanisms are posted price mechanisms.

\footnotetext{
1: Samples: $\left(\hat{v}_{1}, \hat{\sigma}_{1}\right),\left(\hat{v}_{2}, \hat{\sigma}_{2}\right), \ldots,\left(\hat{v}_{m}, \hat{\sigma}_{m}\right)$, where $\hat{\sigma}_{1} \geq \hat{\sigma}_{2} \geq \cdots \geq \hat{\sigma}_{m}$.

2: Parameter: $\epsilon$

3: Input: For each buyer $i$, signal $\sigma_{i}$ (the value $v_{i}$ is unobserved); suppose $\hat{\sigma}_{k_{i}} \geq \sigma_{i} \geq \hat{\sigma}_{k_{i}+1}$.
}

4: Auction:

5: Let $\ell=\Theta\left(\frac{n^{2}}{\epsilon^{4}} \log ^{2} \frac{n}{\epsilon}\right)$.

6: Let $\bar{D}^{i}$ denote the mixture of $D^{\hat{\sigma}_{k_{i}+1}}, D^{\hat{\sigma}_{k_{i}+2}}, \ldots, D^{\hat{\sigma}_{k_{i}+\ell}}$; let $\bar{F}^{i}$ denote the corresponding CDF.

7: For all $i \in[n]$, compute a 2-approximation of $\max _{p: \bar{F}^{i}(p) \leq \frac{1}{2}} p\left(1-\bar{F}^{i}(p)\right)$, denoted as $\mathrm{SREV}_{i}$. (i.e., $\left.\frac{1}{2} \operatorname{SREV}_{i} \leq \max _{p: \bar{F}^{i}(p) \leq \frac{1}{2}} p\left(1-\bar{F}^{i}(p)\right) \leq \operatorname{SREV}_{i}\right)$

8: Let $\mathrm{APX}=\sum_{i=1}^{n} \mathrm{SREV}_{i}$.

9: Let $V=\left\{\frac{2}{\epsilon} \mathrm{Apx}, \frac{2(1-\epsilon)}{\epsilon} \mathrm{APX}, \frac{2(1-\epsilon)^{2}}{\epsilon} \operatorname{ApX}, \ldots, \frac{\epsilon}{n^{2}} \mathrm{APX}, 0\right\}$.

10: For each buyer $i$, round $\hat{v}_{k_{i}+1}{ }^{\epsilon}, \ldots, \hat{v}_{k_{i}+\ell}$ down to the closest value in $V$; let $\hat{v}_{1}^{i}, \ldots, \hat{v}_{\ell}^{i}$ denote the rounded values; let $\bar{E}^{i}$ denote the uniform distribution over $\hat{v}_{1}^{i}, \ldots, \hat{v}_{\ell}^{i}$.

11: Run Myerson auction w.r.t. $\overline{\mathbf{E}}=\bar{E}^{1} \times \cdots \times \bar{E}^{n}$.

Algorithm 4: $\mathrm{M}_{\mathrm{emp}}$, a $1-\mathrm{O}(\epsilon)$ approximately optimal signal auction for multiple agents

the $k_{i}$ in Algorithm 3 is fixed by the end of this stage, and is equal to $j_{i}-1$.)

3. Sample $m+n$ values, $\hat{v}_{j} \sim D^{\hat{\sigma}_{j}}$ for $j \in[m]$ and $v_{i} \sim$ $D^{\sigma_{i}}$ for $i \in[n]$, independently.

In the following discussion, let $\underline{D}^{i}=D^{\sigma_{k_{i}+\ell}}$ with CDF $\underline{F}^{i}$ and let $\underline{\mathbf{D}}=\underline{D}^{1} \times \cdots \times \underline{D}^{n}$. For notation convenience, let $\underline{D}^{i}$ be a point mass at 0 for $i>m$.

LEMMA 12. $\frac{1}{2} \mathrm{OPT}(\underline{\mathbf{D}}) \leq \mathrm{APX} \leq n \mathrm{OPT}(\overline{\mathbf{D}})$, with probability $1-O(\epsilon)$

Proof. The first inequality holds because

$$
\begin{aligned}
\operatorname{SREV}_{i} & \geq \max _{p: \bar{F}^{i}(p) \leq \frac{1}{2}} p\left(1-\bar{F}^{i}(p)\right) & & \left(\mathrm{dfn} \text { of } \mathrm{SREV}_{i}\right) \\
& \geq \max _{p: \underline{F}^{i}(p) \leq \frac{1}{2}} p\left(1-\underline{F}^{i}(p)\right) & & \left(\bar{F}^{i} \succeq \underline{F}^{i}\right) \\
& \geq \frac{1}{2} \max _{p \geq 0} p\left(1-\underline{F}^{i}(p)\right) & & \left(\text { regularity of } \underline{D}^{i}\right)
\end{aligned}
$$

and

$$
\sum_{i \in[n]} \max _{p \geq 0} p\left(1-\underline{F}^{i}(p)\right)=\sum_{i \in[n]} \operatorname{Opt}\left(\underline{D}^{i}\right) \geq \operatorname{Opt}(\underline{\mathbf{D}}) .
$$

The second inequality holds because $\mathrm{SREV}_{i} \leq \mathrm{OpT}(\overline{\mathbf{D}})$.

LEMma 13. With probability $1-O(\epsilon)$, we have

$$
\text { Opt }(\overline{\mathbf{D}}) \geq(1-O(\epsilon)) \text { Opt }(\underline{\mathbf{D}})-O(\epsilon) \text { Opt }(\mathbf{D}) \text {. }
$$

Proof. By Lemma 12 and Lemma 2, truncating $\underline{\mathbf{D}}$ at $\frac{2}{\epsilon} \mathrm{APX} \geq \frac{1}{\epsilon} \mathrm{OPT}(\underline{\mathbf{D}})$ decreases the optimal revenue by at most at $1-O(\epsilon)$ factor. Further, by Lemma 5 rounding values to the closest power of $1-\epsilon$ decreases the optimal revenue by at most another $1-\epsilon$ factor. Finally, by Lemma 12 rounding all values less than $\frac{\epsilon}{n^{2}} \mathrm{APX} \leq \frac{\epsilon}{n} \mathrm{OPT}(\overline{\mathbf{D}})$ to 0 decreases the revenue by at most $\epsilon \operatorname{OpT}(\overline{\bar{D}})$. 
As a simple corollary of the above lemma and Theorem 2 9 we have the following lemma.

LEMMA 14. With probability $1-O(\epsilon)$, we have

$$
\text { Орт }(\overline{\mathbf{E}}) \geq(1-O(\epsilon)) \operatorname{Opt}(\underline{\mathbf{D}})-O(\epsilon) \text { Opт }(\mathbf{D}) \text {. }
$$

Next, we prove the key lemma that lower bounds the expected revenue of the empirical Myerson auction w.r.t. $\overline{\mathbf{E}}$ if we run it on $\mathbf{D}$, conditioned on the realization of signals.

Lemma 15. With probability $1-O(\epsilon)$ (over randomness of the sample values), we have

$$
\begin{aligned}
\mathbb{E}\left[\operatorname{Rev}\left(M_{e m p}, \mathbf{D}\right) \mid \hat{\sigma}_{1}, \ldots, \hat{\sigma}_{m}, \sigma_{1}, \ldots, \sigma_{n}\right] \\
\geq(1-O(\epsilon)) \text { Opt }(\underline{\mathbf{D}})-O(\epsilon) \text { Opt }(\mathbf{D}) .
\end{aligned}
$$

Proof. Let us first explain the proof structure. The lemma follows by Lemma 14 and revenue monotonicity (Theorem 1) if $\mathbf{D}$ stochastically dominates $\overline{\mathbf{E}}$. However, despite the fact that $\mathbf{D}$ stochastically dominates $\overline{\mathbf{D}}$, it does not necessarily dominates $\overline{\mathbf{E}}$ due to randomness in the sample values. To get around this obstacle, we designed for each buyer $i$ a set of random values coupled with the sample values $\hat{v}_{k_{i}+j}, 1 \leq j \leq \ell$, and the uniform distribution over them $E^{i}$ such that (1) E stochastically dominate $\overline{\mathbf{E}}$, and (2) the revenue of any optimal mechanism w.r.t. some distribution over the set of discretized values when we run it on $\mathbf{E}$ is a $1-O(\epsilon)$ approximation of its revenue on $\mathbf{D}$. Then, we can show that the revenue of the empirical Myerson auction (w.r.t. $\overline{\mathbf{E}}$ ) on $\mathbf{D}$ is at least a $1-O(\epsilon)$ fraction of its revenue on $\mathbf{E}$, which is greater than or equal to its revenue on $\overline{\mathbf{E}}$.

Concretely, let $\hat{q}_{j}=\left(1-F^{\hat{\sigma}_{k_{i}+j}}\left(\hat{v}_{k_{i}+j}\right)\right)$, for all $1 \leq j \leq \ell$, be the quantile of $\hat{v}_{k_{i}+j}$ w.r.t. $D^{\hat{\sigma}_{k_{i}+j}}$; let $\tilde{v}_{j}$ be the value with quantile $\hat{q}_{j}$ w.r.t. $D^{i}$. Note that $\hat{q}_{j}$ is uniform from $[0,1]$ over random realization of $\hat{v}_{k_{i}+j}$ conditioned on $\hat{\sigma}_{k_{i}+j}$. So $\tilde{v}_{j}, 1 \leq j \leq \ell$, are i.i.d. samples from $D^{i}$. Let $E^{i}$ be the uniform distribution over $\tilde{v}_{j}, 1 \leq j \leq \ell$.

By our construction and that $D^{i}$ stochastically dominates $D^{\hat{\sigma}_{k_{i}+j}}$ for any $1 \leq j \leq \ell$, we get that $\mathbf{E}$ stochastically dominates $\overline{\mathbf{E}}$. Further, by revenue monotonicity (Theorem 1), we have $\operatorname{REV}\left(M_{\mathrm{emp}}, \mathbf{E}\right) \geq \operatorname{REV}\left(M_{\mathrm{emp}}, \overline{\mathbf{E}}\right)$. Recall that we can alter $\overline{\mathbf{E}}$ such that the optimal auction is unique (Remark 1). Thus, by Theorem 2, we have that $\operatorname{ReV}\left(M_{\mathrm{emp}}, \mathbf{D}\right) \geq(1-$ $O(\epsilon)) \operatorname{Rev}\left(M_{\mathrm{emp}}, \mathbf{E}\right)$. Combining with Lemma 14 completes the proof.

As a simple corollary, we have the following lemma.

LEMma 16. With probability $1-O(\epsilon)$, we have

$$
\begin{aligned}
& \mathbb{E}\left[\operatorname{Rev}\left(M_{\text {emp }}, \mathbf{D}\right)\right] \\
& \quad \geq(1-O(\epsilon)) \mathbb{E}[\operatorname{Opt}(\underline{\mathbf{D}})]-O(\epsilon) \mathbb{E}[\operatorname{Opt}(\mathbf{D})] .
\end{aligned}
$$

Finally, we relate $\mathbb{E}[\mathrm{Opt}(\underline{\mathbf{D}})]$ with $\mathbb{E}[\mathrm{Opt}(\mathbf{D})]$ by proving the following lemma.

LEMma 17. Over the randomness of all three stages, we have

$$
\mathbb{E}[\operatorname{Opt}(\underline{\mathbf{D}})] \geq(1-\epsilon) \mathbb{E}[\operatorname{Opt}(\mathbf{D})]
$$

\footnotetext{
${ }^{9}$ Again, there is an extra complication that the sample values are not iid from the mixture distributions, which can be handled using the approach in Lemma 8.
}

Proof. Relating $\mathbb{E}[$ Opt $(\underline{\mathbf{D}})]$ with $\mathbb{E}[$ Opt $(\mathbf{D})]$ suffices (given Lemma 16). First, consider the expectation over the randomness of the last two stages. Conditioned on the realization of signals $\sigma_{1}^{\prime}, \ldots, \sigma_{m+n}^{\prime}$, over the randomness of the choice of $i_{1}, \ldots, i_{n}$ and random realization of the corresponding values, we can write the expectation of the optimal revenue w.r.t. $\underline{\mathbf{D}}$ as

$$
\frac{1}{\left(\begin{array}{c}
m+n \\
n
\end{array}\right)} \sum_{\left\{i_{1}, \ldots, i_{n}\right\} \subset[m+n]} \text { OPT }\left(D^{\sigma_{i_{1}+\ell}^{\prime}} \times \cdots \times D^{\sigma_{i_{n}+\ell}^{\prime}}\right),
$$

which is at least

$$
\frac{1}{\left(\begin{array}{c}
m+n \\
n
\end{array}\right)} \sum_{\left\{i_{1}, \ldots, i_{n}\right\} \subset\{\ell+1, \ldots, m+n\}} \text { OPT }\left(D^{\sigma_{i_{1}}^{\prime}} \times \cdots \times D^{\sigma_{i_{n}}^{\prime}}\right) .
$$

Next, we consider the expectation of over the randomness of the first stage. Note that $\ell \leq q(\epsilon) m+n$. Taking expectation over random realization of $\sigma_{1}^{\prime}, \ldots, \sigma_{m+n}^{\prime}$, the last expression is lower bounded by

$$
\mathbf{E}_{\sigma_{1}, \ldots, \sigma_{n} \sim F_{\mathcal{D}, \sigma}}\left[\mathbb{1}\left\{\forall i \in[n], F_{\mathcal{D}, \sigma}\left(\sigma_{i}\right) \leq 1-q(\epsilon)\right\} \text { OPT }\left(D^{\sigma_{1}} \times \cdots \times D^{\sigma_{n}}\right)\right] .
$$

By the small tail assumption in signal space (Definition 1), this is at least $(1-\epsilon) \mathbb{E}[$ OPt $(\mathbf{D})]$. So the lemma follows.

Proof Proof of Theorem 5. It follows from Lemma 16 and 17

\subsection{Lower bounds for $n=1$}

TheOREM 6. The sample complexity of any single agent mechanism against any $q$-bounded distribution in the Signals model is

$$
\Omega\left(\frac{\log (1 / q(\epsilon))}{\epsilon^{4}}\right)
$$

Proof. Our instance will have $\frac{\log \frac{1}{\alpha}}{2 \log (1-18 \epsilon)}+1$ conditional distributions. Let $N=\frac{\log \frac{1}{\alpha}}{2 \log (1-18 \epsilon)} \approx \frac{\log \alpha}{\epsilon}$. For the $i$-th conditional distribution (that is the distribution conditioned on signal $\sigma_{i}$ ) we will have two candidate distributions $D_{1}^{\sigma_{i}}$ and $D_{2}^{\sigma_{i}}$, and it will be impossible to extract high revenue from that conditional, without seeing at least $\frac{1}{\epsilon^{3}}$ samples (from that conditional), even when having exact knowledge of the rest of the distributions. The expected revenue from each conditional will be approximately the same, thus, in order for a pricing algorithm to achieve high expected revenue overall, it will have to achieve high expected revenue in most of the conditionals.

Our construction works as follows: for each $i \in[0, N]$ let

$$
c_{i}=\frac{1}{\left(1-2 \epsilon_{0}\right)^{2 i}} .
$$

$D_{1}^{\sigma_{i}}$ is the distribution with c.d.f and p.d.f

$$
\begin{array}{cc}
F_{1}^{i}(x)=1-\frac{c_{i}}{x+c_{i}} & f_{1}^{i}=\frac{c_{i}}{\left(x+c_{i}\right)^{2}} . \\
D_{2}^{\sigma_{i}} \text { has p.d.f. } & \text { if } x \in\left[0, \frac{\left(1-2 \epsilon_{0}\right) c_{i}}{2 \epsilon_{0}}\right] \\
f_{2}^{i}(x)= \begin{cases}\frac{c_{i}}{\left(x+c_{i}\right)^{2}} & \text { if } x>\frac{\left(1-2 \epsilon_{0}\right) c_{i}}{2 \epsilon_{0}} \\
\frac{c_{i}\left(1-2 \epsilon_{0}\right)^{2}}{\left(x-c_{i}\left(1-2 \epsilon_{0}\right)\right)^{2}} & \end{cases}
\end{array}
$$

where $\epsilon_{0}=9 \epsilon$.

Conditioned on seeing signal $\sigma_{i}$ the Myerson revenue is $c_{i}$ for $D_{1}^{\sigma_{i}}$ and $c_{i}\left(1-2 \epsilon_{0}\right)$ for $D_{2}^{\sigma_{i}}$. Notice that both $D_{1}^{\sigma_{i}}$ and $D_{2}^{\sigma_{i}}$ first-order stochastically dominate both $D_{1}^{\sigma_{i-1}}$ and 
$D_{2}^{\sigma_{i-1}}$ (and are dominated by the $D^{\sigma_{i+1}}$ 's). Also, we have that $c_{0}=1$ and $c_{N}=\left(1-2 \epsilon_{0}\right)^{-2 N}=\alpha$, thus the gap between the highest and the lowest revenue is $\alpha$.

In order to make the expected revenue from each conditional approximately the same, we set the probability $p_{i}$ of seeing signal $\sigma_{i}$ equal to $\gamma \cdot \frac{1}{c_{i}}$, where $\gamma$ is the normalizing factor, i.e. $\gamma=\frac{1}{\sum_{j=0}^{N} \frac{1}{c_{j}}}$.

The optimal expected revenue is upper bounded by:

$$
O P T=\sum_{i=0}^{N} p_{i} \text { OPT }\left(D^{\sigma_{i}}\right) \leq \sum_{i=0}^{N} c_{i} p_{i}=\gamma(N+1)
$$

Dropping $p_{N}$ in probability mass in signal space results in dropping about $\gamma$ in terms of revenue, i.e. $\frac{1}{N}$ of the total revenue. Dropping $p_{N}+p_{N-1}$ results in dropping a $\frac{2}{N}$ fraction of the total revenue, and so on; thus, in order to drop an $\epsilon$ fraction of the total revenue, and since $N$ is approximately $\frac{\log \alpha}{\epsilon}$, we need to drop $\sum_{i=N-\log \alpha}^{N} p_{i}$ in probability. This simplifies to

$$
\left(\frac{(1-2 \epsilon)}{4 \epsilon(1-\epsilon)}\right)^{2} \cdot \frac{1}{\alpha} \cdot \frac{1}{\alpha^{2 \log (1-2 \epsilon)}} .
$$

Using this, we can get the following relation between $q(\epsilon)$ and $\alpha: \log \alpha \approx \log \left(\frac{1}{q(\epsilon)}\right)$.

We omit the proof of the following claim:

Claim 6. Any pricing algorithm that is $(1-\epsilon)$ approximate for this instance must be $(1-3 \epsilon)$ approximate for at least half of the signals.

We will proceed to show that in order to $(1-3 \epsilon)$ approximate the revenue for a given signal $\sigma_{i}$, any pricing algorithm requires $\Omega\left(\frac{1}{\epsilon^{3}}\right)$ samples from that signal. Thus, since any $(1-\epsilon)$ approximate pricing algorithm requires $\Omega\left(\frac{1}{\epsilon^{3}}\right)$ approximate in at least $\frac{N+1}{2}>\frac{\log \frac{1}{\alpha}}{4 \log (1-18 \epsilon)}$ of the signals, the total number of signals required is $\Omega\left(\frac{\log \frac{1}{\alpha}}{2 \log (1-18 \epsilon)} \frac{1}{\epsilon^{3}}\right)$, which is $\Omega\left(\frac{\log \alpha}{\epsilon^{4}}\right)$, and thus, since $\log \alpha \approx \log \left(\frac{1}{q(\epsilon)}\right)$ we get the desired lower bound for $q$-bounded distributions.

LEMMA 18. Any pricing algorithm that is $(1-3 \epsilon)$ approximate for both $D_{1}^{\sigma_{i}}$ and $D_{2}^{\sigma_{i}}$ requires $\Omega\left(\frac{1}{\epsilon^{3}}\right)$ samples from the distribution conditioned on $\sigma_{i}$.

Proof. First, notice that even exact knowledge of the distributions conditioned on the rest of the signals does not help here; we're back to a one-distribution problem.

We will follow a similar approach to [12] we will first bound the KL divergence of $D_{1}^{\sigma_{i}}$ and $D_{2}^{\sigma_{i}}$, then show that the $(1-9 \epsilon)=\left(1-\epsilon_{0}\right)$ optimal price sets are disjoint. We state here, without proofs, a theorem and a lemma from 12 that we use:

TheOREM 7 (THeOREM 4.2 From [12]). If value distributions $D_{1}$ and $D_{2}$ have disjoint $\left(1-3 \epsilon^{\prime}\right)$ approximate price sets, and there is a pricing algorithm that is $\left(1-\epsilon^{\prime}\right)$ approximate for both $D_{1}$ and $D_{2}$, then the algorithm uses at least $4 / 9\left(D_{K L}\left(D_{1} \| D_{2}\right)+D_{K L}\left(D_{2} \| D_{1}\right)\right)^{-1}$ samples.

Lemma 19 (LEMma 4.4 FROM 12 ). If value distributions $D_{1}$ and $D_{2}$ with densities $f_{1}$ and $f_{2}$ satisfy that

$$
\left(1+\epsilon^{\prime}\right)^{-1} \leq \frac{f_{1}(\omega)}{f_{2}(\omega)} \leq\left(1+\epsilon^{\prime}\right)
$$

for every $\omega \in \Omega$, and there is a subset of outcomes $\Omega^{\prime}$ such that $p_{1}(\omega)=p_{2}(\omega)$ for every $\omega \in \Omega^{\prime}$, then

$$
D_{K L}\left(D_{1} \| D_{2}\right)+D_{K L}\left(D_{2} \| D_{1}\right) \leq\left(\epsilon^{\prime}\right)^{2}\left(1-p_{1}\left(\Omega^{\prime}\right)\right)
$$

Notice that:

$$
\frac{f_{1}^{i}}{f_{2}^{i}}= \begin{cases}1 & \text { if } x \in\left[0, \frac{\left(1-2 \epsilon_{0}\right) c_{i}}{2 \epsilon_{0}}\right] \\ \frac{c_{i}}{c_{i}\left(1-2 \epsilon_{0}\right)^{2}} \cdot \frac{\left(x-c_{i}\left(1-2 \epsilon_{0}\right)\right)^{2}}{\left(x+c_{i}\right)^{2}} & \text { if } x>\frac{\left(1-2 \epsilon_{0}\right) c_{i}}{2 \epsilon_{0}}\end{cases}
$$

Thus it holds that $\frac{f_{1}^{i}}{f_{2}^{i}} \in\left[\left(1-2 \epsilon_{0}\right)^{2},\left(1-2 \epsilon_{0}\right)^{2}\right]$, and thus $\frac{f_{1}^{i}}{f_{2}^{i}} \in\left[\left(1+\frac{\epsilon_{0}}{1-2 \epsilon_{0}}\right)^{-1},\left(1+\frac{\epsilon_{0}}{1-2 \epsilon_{0}}\right)\right]$.

$D_{1}^{\sigma_{i}}$ and $D_{2}^{\sigma_{i}}$ are identical for $x \leq \frac{\left(1-2 \epsilon_{0}\right) c_{i}}{2 \epsilon_{0}}$. In quantile space, this corresponds to $q \geq 2 \epsilon_{0}$. Thus, by applying Lemma 19 we have that:

$$
\begin{aligned}
D_{K L}\left(D_{1}^{\sigma_{i}} \| D_{2}^{\sigma_{i}}\right)+ & D_{K L}\left(D_{2}^{\sigma_{i}} \| D_{1}^{\sigma_{i}}\right) \\
& \leq\left(\frac{\epsilon_{0}}{1-2 \epsilon_{0}}\right)^{2} 2 \epsilon_{0}=\frac{2 \epsilon_{0}^{3}}{\left(1-2 \epsilon_{0}\right)^{2}}
\end{aligned}
$$

Moreover, it's easy to see that the $\left(1-\epsilon_{0}\right)$ price sets are disjoint, by comparing the revenue as a function of the price. By combining equation 7 with this observation and Theo$\operatorname{rem} 7\left(\epsilon^{\prime}=3 \epsilon\right)$ we get the desired bound.

\section{EXTENSIONS}

In this section, we will explain how to use the techniques developed in this paper to show sample complexity upper bounds for several natural extensions of the settings discussed in the main text. Next, we will present a brief introduction of these extensions. For the key differences in the algorithm and the analysis of the extensions compared to those in the main text see the full version of this paper.

\section{Matroid Constraint.}

In this setting, the seller may serve a subset of buyers $S$ that is independent w.r.t. to a matroid (e.g., [1]). Let $\mathbf{M}$ denote the set of subsets of buyers that can be served simultaneously. The key property that we use about matroid constraint is that the VCG mechanism with duplicates is a 2approximation for regular value distributions under matroid constraint 11 .

\section{Downward-closed Constraint.}

In this setting, the subsets of buyers that the seller can serve simultaneously is downward-closed, i.e., if serving a subset of buyers $S$ is feasible then serving any subsets of $S$ is also feasible. We will assume without loss of generality that serving any single buyer is feasible. Again, let $\mathbf{M}$ denote the set of subsets of buyers that can be served simultaneously. We will show that Lemma 2 generalizes to any singleparameter mechanism design problem with downward-closed constraint and regular value distributions. The proof is deferred to the full version of this paper.

Lemma 20 (Generalized Lemma 2). Consider any downward closed constraint M. Suppose $\frac{1}{4} \geq \epsilon>0$ is a constant. Suppose $\bar{v} \geq \frac{1}{\epsilon} \operatorname{OpT}(\mathbf{D})$. Let $\bar{D}^{1}, \ldots, \bar{D}^{n}$ be the distributions obtained by truncating $D^{1}, \ldots, D^{n}$ at $\bar{v}$, i.e., a 
sample $\bar{v}_{i}$ from $\bar{D}^{i}$ is obtained by first sampling $v_{i}$ from $D^{i}$ and then letting $\bar{v}_{i}=\min \left\{v_{i}, \bar{v}\right\}$. Then, we have

$$
\operatorname{Opt}\left(\Pi_{i} \bar{D}^{i}\right) \geq(1-4 \epsilon) \operatorname{Opt}(\mathbf{D})
$$

\section{Proof sketch of Lemma 20}

Let $\bar{q}_{i}=1-F(\bar{v})$ denote the quantile of value $\bar{v}$ w.r.t. $D^{i}$. Let $\phi_{i}\left(q_{i}\right)$ and $\bar{\phi}_{i}\left(q_{i}\right)$ denote the virtual value at quantile $q_{i}$ w.r.t. $D^{i}$ and $\bar{D}^{i}$ respectively. Then,

$$
\bar{\phi}_{i}\left(q_{i}\right)= \begin{cases}\phi_{i}\left(q_{i}\right) & \text { if } \bar{q}_{i}<q_{i} \leq 1 ; \\ \bar{v} & \text { if } 0 \leq q_{i} \leq \bar{q}_{i} .\end{cases}
$$

We first develop the following upper bounds on $\bar{q}_{1}, \ldots, \bar{q}_{n}$. The omitted proofs are in the full version 6].

Lemma 21. For any $i \in[n]$, we have $\bar{q}_{i} \leq \epsilon$.

LEMma 22. The probability that at least one buyer has value greater than or equal to $\bar{v}$ is at most $\epsilon$ and is at least $(1-\epsilon) \sum_{i \in[n]} \bar{q}_{i}$, i.e.,

$$
\epsilon \geq 1-\prod_{i \in[n]}\left(1-\bar{q}_{i}\right) \geq(1-\epsilon) \sum_{i \in[n]} \bar{q}_{i}
$$

PROOF. If the mechanism serves at most one buyer and runs the second-price auction with reserve price $\bar{v}$ gets revenue $\left(1-\prod_{i \in[n]}\left(1-\bar{q}_{i}\right)\right) \bar{v}$, which must be less than or equal to the optimal revenue Opt $(\mathbf{D})$. Since $\bar{v} \geq \frac{1}{\epsilon}$ Opt $(\mathbf{D})$, we get that $1-\prod_{i \in[n]}\left(1-\bar{q}_{i}\right) \leq \epsilon$. As a result, we get that $\prod_{i \in[n]}\left(1-\bar{q}_{i}\right) \geq 1-\epsilon$. Therefore, we have

$$
\begin{aligned}
1-\prod_{i \in[n]}\left(1-\bar{q}_{i}\right) & =\sum_{i \in[n]} \bar{q}_{i} \prod_{j \neq i}\left(1-\bar{q}_{j}\right) \\
& \geq \sum_{i \in[n]} \bar{q}_{i} \prod_{j \in[n]}\left(1-\bar{q}_{j}\right) \geq(1-\epsilon) \sum_{i \in[n]} \bar{q}_{i}
\end{aligned}
$$

Note that both $D^{i}$ and $\bar{D}^{i}$ are regular. Myerson auction simply serves a subset of the buyers that maximizes the virtual surplus. So we have (recall that $\mathbf{M}$ is the set of independent sets of the matroid)

$$
\begin{gathered}
\operatorname{Opt}(\mathbf{D})=\int_{[0,1]^{n}} \max _{S \in \mathbf{M}} \sum_{i \in S} \phi_{i}\left(q_{i}\right) d^{n} \mathbf{q}, \quad \text { and } \\
\operatorname{Opt}(\overline{\mathbf{D}})=\int_{[0,1]^{n}} \max _{S \in \mathbf{M}} \sum_{i \in S} \bar{\phi}_{i}\left(q_{i}\right) d^{n} \mathbf{q}
\end{gathered}
$$

Given a quantile vector $\mathbf{q}$, let $H(\mathbf{q})=\left\{i \in[n]: q_{i} \leq \bar{q}_{i}\right\}$ denote the buyers whose values are higher than $\bar{v}$; let $L(\mathbf{q})=$ $[n] \backslash H(\mathbf{q})$ denote the set of buyers whose values are smaller than $\bar{v}$. We can divide the revenue of Myerson's optimal auction into two parts, the revenue from high value buyers and one from low value buyers. We will upper bound them separately.

$$
\begin{aligned}
\operatorname{Opt}(\mathbf{D}) \leq \int_{[0,1]^{n}} \max _{S \in H(\mathbf{q})} & \sum_{i \in S} \phi_{i}\left(q_{i}\right) d^{n} \mathbf{q} \\
& +\int_{[0,1]^{n}} \max _{S \in L(\mathbf{q})} \sum_{i \in S} \phi_{i}\left(q_{i}\right) d^{n} \mathbf{q}
\end{aligned}
$$

We will also divide the revenue of Myerson's optimal auction w.r.t. the truncated distributions into two parts, the revenue when there are at least one high value buyer, and the revenue when all buyers have low values.

$$
\begin{aligned}
\operatorname{Opt}(\overline{\mathbf{D}})=\int_{[0,1]^{n} \backslash \prod_{j}\left[\bar{q}_{j}, 1\right]} & \max _{S \in[n]} \sum_{i \in S} \bar{\phi}_{i}\left(q_{i}\right) d^{n} \mathbf{q} \\
& +\int_{\prod_{j}\left[\bar{q}_{j}, 1\right]} \max _{S \in[n]} \sum_{i \in S} \bar{\phi}_{i}\left(q_{i}\right) d^{n} \mathbf{q}
\end{aligned}
$$

It suffices to show the following two inequalities, the proofs of which can be found in the full version 6 of this paper.

$$
\begin{aligned}
\int_{[0,1]^{n} \backslash \prod_{j}\left[\bar{q}_{j}, 1\right]} \max _{S \in[n]} \sum_{i \in S} \bar{\phi}_{i}\left(q_{i}\right) d^{n} \mathbf{q} \\
\geq(1-2 \epsilon) \int_{[0,1]^{n}} \max _{S \in H(\mathbf{q})} \sum_{i \in S} \phi_{i}\left(q_{i}\right) d^{n} \mathbf{q} \\
\int_{\prod_{j}\left[\bar{q}_{j}, 1\right]} \max _{S \in[n]} \sum_{i \in S} \bar{\phi}_{i}\left(q_{i}\right) d^{n} \mathbf{q} \\
\geq(1-2 \epsilon) \int_{[0,1]^{n}} \max _{S \in L(\mathbf{q})} \sum_{i \in S} \phi_{i}\left(q_{i}\right) d^{n} \mathbf{q}
\end{aligned}
$$

\section{VCG with Reserves.}

It is also natural to consider simpler classes of mechanisms such as the VCG mechanism with individual reserve prices considered by Hartline and Roughgarden [11]. We may ask what is the number of samples sufficient/necessary to estimate a set of reserve prices that obtain a $1-\epsilon$ fraction of the optimal revenue within this family of mechanisms.

\subsection{Non-iid Model}

\section{Single-parameter problem under Matroid constraint.}

The algorithm, the sample complexity analysis in Section 4 and the same upper bound of $O\left(n \epsilon^{-4} \log ^{2} \frac{n}{\epsilon}\right)$ generalize straightforwardly to this setting, noting that the VCG mechanism with duplicates is a 2-approximation not only for selling a single item but also for any single parameter problem in this setting, and that Lemma 2 generalizes (i.e., Lemma 20.

\section{Single-parameter under downward-closed constraint.}

The algorithm and the analysis for this case are essentially the same as the matroid case, with only one caveat: it is not clear whether the VCG mechanism with duplicates is a constant factor approximation. Thus, we will resort to SREV in Algo 1 instead of APX as our coarser estimation of the optimal revenue, and change the set of discretized values in Algo 2 to $V=\left\{\frac{2 \operatorname{SREV}}{\epsilon}, \frac{2(1-\epsilon) \operatorname{SReV}}{\epsilon}, \frac{2(1-\epsilon)^{2} \mathrm{SREV}}{\epsilon}, \ldots, \frac{\epsilon \operatorname{SREV}}{n^{2}}\right\}$ accordingly. Note that SREV is only an $n$-approximation. As a result, the largest discretized value could be up to $\frac{n}{\epsilon}$ times larger than the expected revenue (in contrast, the largest discretized value is only $O\left(\frac{1}{\epsilon}\right)$ times larger than the expected revenue in the matroid case). Thus, the number of samples needed to estimate the expected revenue according to Bernstein's inequality is $O(n)$ times larger than that in the matroid case. 


\section{$V C G$ with Reserves.}

The algorithm and the analysis follows the same framework as those for Myerson's auction in Section 4. The only difference in the algorithm is that instead of running Myerson's auction w.r.t. the empirical product distribution $\mathbf{E}$ (line 6 of Algorithm 2), we will find the best reserve prices that maximize the revenue of VCG mechanism with reserves. It is not clear how to implement this step in polynomial time so our sample complexity bound for this case is information theoretic. To get computational efficiency, it suffices to design a poly-time algorithm that computes the best reserve prices when the number of candidate prices is polynomial. We leave this as an interesting future direction.

The sample complexity upper bound we can prove for VCG with reserves is $O\left(n \epsilon^{-3} \log \frac{n}{\epsilon}\right)$, which is better than the bound for Myerson's auction. The improvement comes from the fact that given a set $V$ of finitely many prices, the number of different VCG mechanisms with reserves is only $|V|^{n}$; in contrast, the number of different Myerson's auction is roughly $(|V| n)^{|V| n}$ as shown in Section 4. Recall that the sample complexity upper bound using our approach is proportional to the logarithm of the number of candidate mechanisms. Thus, the sample complexity upper bound we get for VCG with reserves is $|V| \approx \tilde{O}\left(\frac{1}{\epsilon}\right)$ times better than that of Myerson's auction.

\subsection{Signal Model}

The sample complexity bounds of the extended settings in the signal model follow directly by the results in the previous subsection and the techniques in Section 5(i.e., for each buyer, use the sample values corresponding to the $\ell$ signals closest to the buyer's signal from below in place of the actual samples from the buyer's prior, and run the algorithm in the non-iid model). Note that in the signal model, we need to estimate the optimal revenue w.r.t. a mixture of regular distributions, which is not necessarily regular. Thus, unlike the non-iid model, we will not be able to use the VCG mechanism with duplicates to get a better approximation of the optimal revenue and better sample complexity upper bounds even if the feasibility constraint is a matroid. Therefore, we have the same sample complexity upper bound for selling a single item, matroid constraint, and downward-closed constraint.

\section{ACKNOWLEDGEMENTS}

We would like to thank Matt Weinberg for the useful discussions about Revenue Monotonicity.

\section{REFERENCES}

[1] P. Azar, C. Daskalakis, S. Micali, and S. M. Weinberg. Optimal and efficient parametric auctions. In Proc. of ACM-SIAM SODA, pages 596-604, 2013.

[2] M.-F. Balcan, A. Blum, J. D. Hartline, and Y. Mansour. Reducing mechanism design to algorithm design via machine learning. Journal of Computer and System Sciences, 74(8):1245-1270, 2008.

[3] S. Bhattacharya, E. Koutsoupias, J. Kulkarni, S. Leonardi, T. Roughgarden, and X. Xu. Near-optimal multi-unit auctions with ordered bidders. In Proc. of ACM EC, pages 91-102, 2013.

[4] J. Bulow and P. Klemperer. Auction versus negotiations. American Economic Review, 1996.
[5] R. Cole and T. Roughgarden. The sample complexity of revenue maximization. In Proc. of ACM STOC, pages 243-252, 2014.

[6] N. R. Devanur, Z. Huang, and C.-A. Psomas. The Sample Complexity of Auctions with Side Information. ArXiv e-prints, Nov. 2015.

[7] P. Dhangwatnotai, T. Roughgarden, and Q. Yan. Revenue maximization with a single sample. Games and Economic Behavior, 2014.

[8] S. Dughmi, L. Han, and N. Nisan. Sampling and representation complexity of revenue maximization. In Web and Internet Economics, pages 277-291. Springer, 2014.

[9] E. Elkind. Designing and learning optimal finite support auctions. In Proc. of ACM-SIAM SODA, pages 736-745, 2007.

[10] E. Even-Dar, J. Feldman, Y. Mansour, and S. Muthukrishnan. Position auctions with bidder-specific minimum prices. In Internet and Network Economics, pages 577-584. Springer, 2008.

[11] J. D. Hartline and T. Roughgarden. Simple versus optimal mechanisms. In Proceedings of the 10th ACM conference on Electronic commerce, pages 225-234. ACM, 2009.

[12] Z. Huang, Y. Mansour, and T. Roughgarden. Making the most of your samples. In Proceedings of the Sixteenth ACM Conference on Economics and Computation, EC '15, Portland, OR, USA, June 15-19, 2015, pages 45-60, 2015.

[13] S. Lahaie and D. M. Pennock. Revenue analysis of a family of ranking rules for keyword auctions. In Proceedings of the 8th ACM conference on Electronic commerce, pages 50-56. ACM, 2007.

[14] S. Leonardi and T. Roughgarden. Prior-free auctions with ordered bidders. In Proceedings of the Forty-fourth Annual ACM Symposium on Theory of Computing, STOC '12, pages 427-434, New York, NY, USA, 2012. ACM.

[15] J. Morgenstern and T. Roughgarden. The pseudo-dimension of nearly-optimal auctions. In NIPS, page Forthcoming, 122015.

[16] R. B. Myerson. Optimal auction design. Mathematics of Operations Research, 6(1):58-73, 1981.

[17] M. Ostrovsky and M. Schwarz. Reserve prices in internet advertising auctions: A field experiment. In Proceedings of the 12th ACM conference on Electronic commerce, pages 59-60. ACM, 2011.

[18] B. Roberts, D. Gunawardena, I. A. Kash, and P. Key. Ranking and tradeoffs in sponsored search auctions. In Proceedings of the fourteenth ACM conference on Electronic commerce, pages 751-766. ACM, 2013.

[19] M. Sion et al. On general minimax theorems. Pacific J. Math, 8(1):171-176, 1958.

[20] B. Sivan and V. Syrgkanis. Vickrey auctions for irregular distributions. In $\mathrm{Web}$ and Internet Economics, pages 422-435. Springer, 2013.

[21] D. R. Thompson and K. Leyton-Brown. Revenue optimization in the generalized second-price auction. In Proceedings of the fourteenth ACM conference on Electronic commerce, pages 837-852. ACM, 2013. 